Subscriber access provided by King Abdullah University of Science and Technology Library

Article

\title{
Functionalization of Strongly Interacting Magnetic Nanocubes with (Thermo)responsive Coating and their Application in Hyperthermia and Heat-Triggered Drug Delivery \\ Hamilton Kakwere, Manuel Pernia Leal, Maria-Elena Materia, Alberto Curcio, Pablo Guardia, Dina Niculaes, Roberto Marotta, Andrea Falqui, and Teresa Pellegrino
}

ACS Appl. Mater. Interfaces, Just Accepted Manuscript • DOI: 10.1021/am5088117 • Publication Date (Web): 03 Apr 2015

Downloaded from http://pubs.acs.org on April 7, 2015

\section{Just Accepted}

"Just Accepted" manuscripts have been peer-reviewed and accepted for publication. They are posted online prior to technical editing, formatting for publication and author proofing. The American Chemical Society provides "Just Accepted" as a free service to the research community to expedite the dissemination of scientific material as soon as possible after acceptance. "Just Accepted" manuscripts appear in full in PDF format accompanied by an HTML abstract. "Just Accepted" manuscripts have been fully peer reviewed, but should not be considered the official version of record. They are accessible to all readers and citable by the Digital Object Identifier (DOI®). "Just Accepted" is an optional service offered to authors. Therefore, the "Just Accepted" Web site may not include all articles that will be published in the journal. After a manuscript is technically edited and formatted, it will be removed from the "Just Accepted" Web site and published as an ASAP article. Note that technical editing may introduce minor changes to the manuscript text and/or graphics which could affect content, and all legal disclaimers and ethical guidelines that apply to the journal pertain. ACS cannot be held responsible for errors or consequences arising from the use of information contained in these "Just Accepted" manuscripts. 


\section{Functionalization of Strongly Interacting Magnetic Nanocubes with (Thermo)responsive Coating and their} Application in Hyperthermia and Heat-Triggered Drug

\section{Delivery}

Hamilton Kakwere ${ }^{a}$, Manuel Pernia Leal ${ }^{a}$, Maria Elena Materia ${ }^{a}$, Alberto Curcio ${ }^{a}$, Pablo Guardia $^{a}$, Dina Niculaes $^{a}$, Roberto Marotta $^{a}$, Andrea Falqui $^{a, b}$ and Teresa Pellegrino ${ }^{a}$ *

a Istituto Italiano di Tecnologia, via Morego 30, 16143, Genova, Italy

b King Abdullah University of Science and Technology (KAUST), BESE division, Thuwal, Kingdom of Saudi Arabia.

E-mail: teresa.pellegrino@iit.it

Keywords: Smart materials, cubic magnetic nanoparticles, thermo-responsive, pH-responsive, drug release, hyperthermia

Abstract: Herein we prepare nanohybrids by incorporating iron oxide nanocubes (cubic-IONPs) within a thermo-responsive polymer shell that can act as drug carriers for doxorubicin(doxo). The cubic-shaped nanoparticles employed are at the interface between superparamagnetic and ferromagnetic behavior and have an exceptionally high specific absorption rate (SAR) but their functionalization is extremely challenging compared to bare superparamagnetic iron oxide nanoparticles as they strongly interact with each other. By conducting the polymer grafting reaction using reversible addition-fragmentation chain transfer (RAFT) polymerization in a viscous solvent medium, we have here developed a facile approach to decorate the nanocubes with stimuli-responsive polymers. When the thermo-responsive shell is composed of poly( $\mathrm{N}$-isopropyl acrylamide-co-polyethylene glycolmethylether acrylate), nanohybrids have a phase transition temperature, the lower critical solution temperature (LCST), above $37{ }^{\circ} \mathrm{C}$ in physiological conditions. Doxo loaded nanohybrids exhibited a negligible drug release below $37{ }^{\circ} \mathrm{C}$ but showed a consistent release of their cargo on demand by exploiting the capability of the nanocubes to generate heat under an alternating magnetic field (AMF). Moreover, the drug free nanocarrier does not 
exhibit cytotoxicity even when administered at high concentration of nanocubes $(1 \mathrm{~g} / \mathrm{L}$ of iron) and internalized at high extent (260 pg of iron per cell). We have also implemented the synthesis protocol to decorate the surface of nanocubes with poly(vinylpyridine) polymer and thus prepare pH-responsive shell coated nanocubes.

\section{Introduction}

Emerging technologies in preparation of magnetic nanoparticles have afforded materials with a wide range of potential applications including magnetic resonance imaging (MRI) agents, carriers for drug/gene delivery, hyperthermia agents and biosensors. ${ }^{1-4}$ Non-aqueous synthesis protocols allow for the preparation of iron oxide nanoparticles (IONPs) with control over the crystallinity and thus obtaining particles with desirable magnetic properties for the distinct applications. ${ }^{5}$ However, these methods yield hydrophobic nanoparticles capped with organic surfactants which hinder their direct application in biomedicine. ${ }^{4,6}$ With the persistent developments in synthesis of magnetic nanoparticles/IONPs targeted for various biologic applications, there is accordingly the need to continuously develop strategies for their functionalization.

Functionalization of IONPS has been conducted via modification of the IONPs surfaces with small molecules as well as with polymers. ${ }^{7}$ The combination of polymers, specifically stimuli-responsive polymers, with inorganic particles represents a fascinating route for generation of new types of materials and devices integrating properties of inorganic particles with those of polymers. ${ }^{8}$ The main strategies employed for polymer functionalization of IONPs include the grafting from and grafting to techniques ${ }^{6,9}$ which lately have been employed in combination with living radical polymerization (LRP) techniques. ${ }^{10-11}$ LRP techniques allow rapid access to well-defined functional (co)polymers of varying molecular architecture and thus offer good control over the size of hybrid nanostructures. ${ }^{12}$ Herein, we report a potentially generic and facile approach for the functionalization of strongly interacting cubic shaped IONPS (cubic-IONPS) via the grafting from approach using an LRP technique (reversible additionfragmentation chain transfer (RAFT)) polymerization. ${ }^{13}$ Although several reports on functionalization of spherical-IONPs (mainly prepared by co-precipitation methods) via RAFT polymerization and other LRP techniques have demonstrated the feasibility to synthesize thermo-responsive shell on top of IONPs, ${ }^{14-}$ ${ }^{18,19-20}$ the protocols previously developed have mainly used nanoparticles showing superparamagnetic behavior at room temperature. These nanoparticles are, by their nature, non-interacting particles, as they miss any remanence magnetization at room temperature and in absence of an external magnetic 
field. Very recently, our group has reported the non-hydrolytic synthesis of 19-22 nm cubic-IONPs having a magnetic behavior at the interface between the superparamagnetic and ferromagnetic regime at room/body temperature. In a comparative study, we have observed that cubic-IONPs with sizes of 19-22 $\mathrm{nm}$ have the best heating performance as they show very high specific absorption rates (SAR) values whilst above or below this nanoparticle size range, the SAR values decreases and the heating performance becomes lower. This makes the 19-22 nm cubic-IONPs very promising for hyperthermia applications. ${ }^{21}$ Further, they are composed of biocompatible species (only iron and oxygen) and at the same time, they exhibited efficient heat dissipation at frequency and field conditions which are applicable to patients. ${ }^{22}$ Given the high SAR values, lower doses of cubic-IONPs could be applied in vivo for reaching therapeutic temperatures. ${ }^{23}$ Furthermore, in vivo degradation in liver of those iron oxide nanocubes has been reported by some of us. ${ }^{24}$ However, because of their size and magnetic properties those cubic-IONPs strongly interact with each other and many challenges are encountered when trying to functionalize such nanoparticles. ${ }^{9,} 11$ Amphiphilic polymer enwrapping and ligand exchange protocols with pegylated molecules have been adapted to allow the nanoparticle water transfer. ${ }^{22}$ However, having a functional coating like a thermo-responsive or $\mathrm{pH}$-responsive shell could further enhance their applicability. Herein, using a direct approach, we employ cubic-IONPs as a blueprint of strongly aggregating nanoparticles and we develop protocols at the nanoparticle surface for an in situ polymerization by LRP techniques of responsive polymers: temperature (based on $N$ isopropylacrylamide, NIPAAM) or $\mathrm{pH}$ (based on vinylpyridine,VP). The key to achieve the polymer shell growth on individual cubic-IONPs was the choice of viscous solvents as media in which to perform the polymerization. The thermo-responsive coated cubic-IONPs still preserved their good magnetic properties with the very high SAR values. ${ }^{22}$ The temperature responsive cubic-IONPs were used as drug carriers for chemotherapy. Doxorubicin (doxo) molecules were loaded at room temperature and after drug encapsulation released under an alternating magnetic field (AMF) by exploiting the heating ability of the cubic-IONPs. Under an AMF exposure, the temperature of the thermo-responsive cubic-IONPS increased and the accompanying coil-globule transition of the polymer led to expulsion of the drug from the temperature responsive cubic-IONPs. Such nanostructures may then act as drug carriers, providing a synergistic therapeutic effect in cancer treatment via hyperthermia and chemotherapy with triggered drug release, while not displaying side effects presented by standard chemotherapy. ${ }^{15}$ In this work, we demonstrate that by conducting the surface functionalization of the cubic-IONPs in a viscous medium we can overcome the challenges associated with the functionalization of such strongly interacting nanoparticles and yield hybrid nanomaterials with a potentially wide range of applications. The parallel 
comparison of the implementation of the same procedures to superparamagnetic spherical-IONPS allows us to highlight the challenges we have encountered when using cubic-IONPs.

\section{Results and discussion}

\subsection{The ligand exchange protocol for the preparation of RAFT functionalized nanocubes}

To functionalize the cubic-IONPs with the responsive polymer, we opted for the grafting from approach using RAFT polymerization taking into account the high grafting densities that arise from using this grafting approach and the versatility of the polymerization technique. Following procedures similar to those employed in literature for the functionalization of superparamgnetic IONPs, we first synthesized a catechol bearing RAFT agent (CTCLRA) to act as an anchor for the polymer chains on the surface as the catechol moiety is known to bind strongly to iron oxide surface., 25 The CTCLRA was obtained in good yield $(87 \%)$ by a two-step reaction between dopamine and succinimide-activated RAFT agent and the structure was confirmed by ${ }^{1} \mathrm{H}$ NMR (Figure 1) (for the characterization of the intermediate compound see Figure S1 of the supporting information, SI). Subsequently, the oleic acid capped cubic-IONPs in $\mathrm{CHCl}_{3}$ were exchanged with the CTCLRA using a large excess of RAFT agent (the initial mixing ratio was fixed at 16 RAFT agent molecules $/ \mathrm{nm}^{2}$ ) in tetrahydrofuran (THF). It is worth mentioning that we have also observed that the ligand exchange process with the RAFT agent, the CTCLRA, could also be conducted by adding the RAFT agent dissolved in THF directly to the crude reaction mixture obtained at the end of the synthesis procedure of the nanocubes (usually dissolved in squalane/dibenzyl ether). ${ }^{22} \mathrm{At}$ the end of the synthesis of the cubic-IONPs, the reaction mixture was allowed to cool to $60{ }^{\circ} \mathrm{C}$ and without dismantling the apparatus, under argon, the RAFT agent solution in THF was added and the ligand exchange process was left to proceed overnight at room temperature. The CTCLRA-coated nanocubes could be then recovered by centrifugation and be completely resuspended in THF. Also, the CTCLRA exchanged nanocubes were insoluble in hexane which was a good solvent for the starting nanoparticles thus indicating the change in the coating at their surface.

The success of the ligand exchange protocol was probed by FTIR and elemental analysis. After CTCLRA exchange, the amide $\mathrm{N}-\mathrm{H}$ stretch at $3400 \mathrm{~cm}^{-1}$ in the spectrum of the CTCLRA coated nanoparticles reveal the presence of the RAFT agent at the nanoparticle surface (this peak was absent in the spectrum of the starting nanoparticles) (Figure 1). Furthermore, the sulfur element which was absent in the as- 
synthesized nanocubes (based on ICP) was abundantly detected via elemental analysis only on CTCLRA coated IONPs after ligand exchange, indicating the successful attachment of the sulfur bearing CTCLRA. Based on the sulfur and iron content measured by ICP, the grafting density was estimated to be 4 molecules/nm².

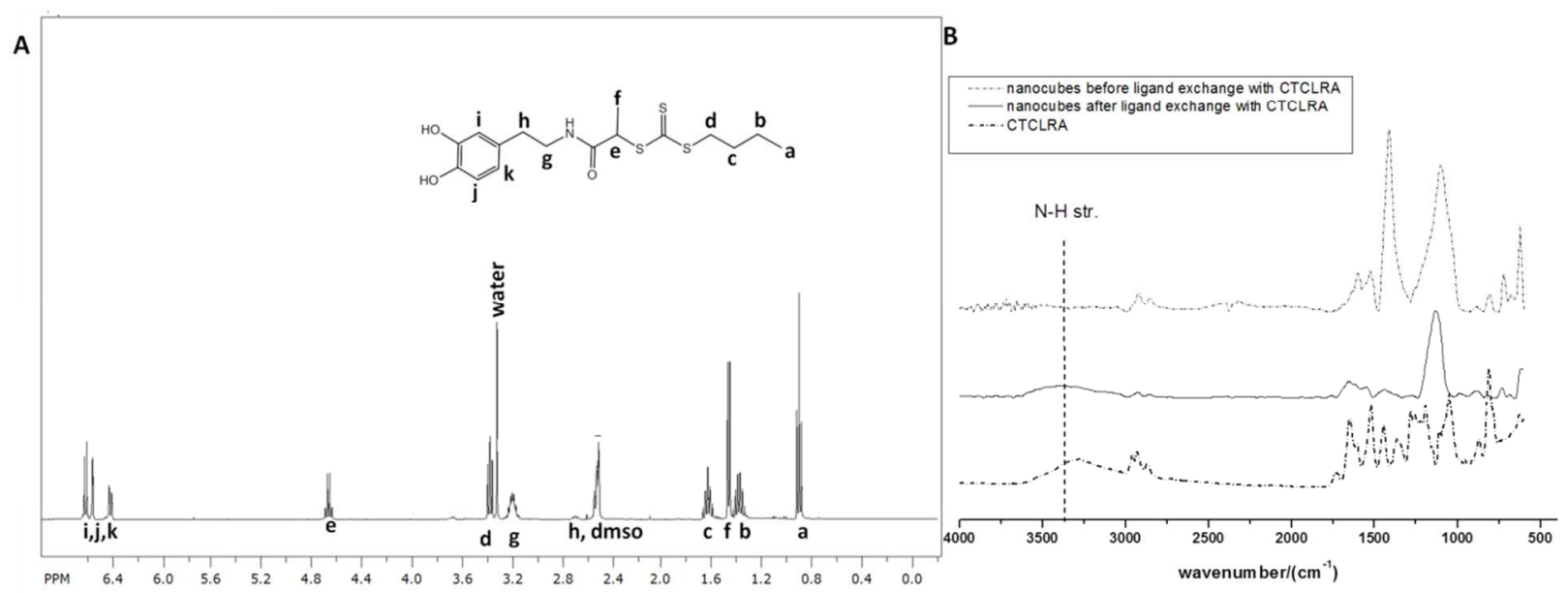

Figure 1: (A) ${ }^{1} \mathrm{H}$ NMR spectrum of catechol functionalized RAFT agent (CTCLRA) in DMSO- $d_{6}$ and (B)FTIR spectra of CTCLRA and nanocubes before and after ligand exchange with CTCLRA.

\subsection{Polymerization reaction of thermo-responsive shell on the RAFT-functionalized nanocubes}

After functionalizing the cubic-IONPs with CTCLRA, we adopted an approach similar to that employed by Ohno et al. $^{26}$ to grow polymer on superparamagnetic nanoparticles for the functionalization of our strongly interacting cubic-IONPs with poly( $N$-isopropylacrylamide) (PNIPAAM) which is a non-toxic polymer with a lower critical solution temperature (LCST) of about $32{ }^{\circ} \mathrm{C}$ in water. ${ }^{27}$ Although the transition temperature of PNIPAAM is lower than $37{ }^{\circ} \mathrm{C}$, copolymerizing NIPAAM with hydrophilic/hydrophobic monomers allows tuning of the LCST. However, under the polymerization conditions employed for superparamagnetic nanoparticles and in THF (see the section of the material and methods) the reaction did not give the desired colloidally stable nanohybrids and polymer coated nanocubes precipitated out of solution within the first few minutes of polymerization. Functionalization under continuous stirring on a magnetic stirrer was not possible since the cubic-IONPs are strongly attracted to magnetic surfaces and thus they do not stay dispersed but instead they "rush" to the magnet and clump up at the bottom of the reaction vessel. Functionalization was however possible 
when the reaction was carried out in an ultrasonication bath under continuous sonication. The samples obtained under continuous sonication appeared mostly as clusters of nanocubes as observed by Transmission Electron Microscope (TEM) analysis (Figure 2E) rather than being singly coated cubic-IONPs as observed upon functionalization of spherical and superparamagnetic IONPs by Ohno et. al (see also Figure $2 \mathrm{G}) .{ }^{26}$ suggesting that in our case, particle aggregation took place under these conditions.

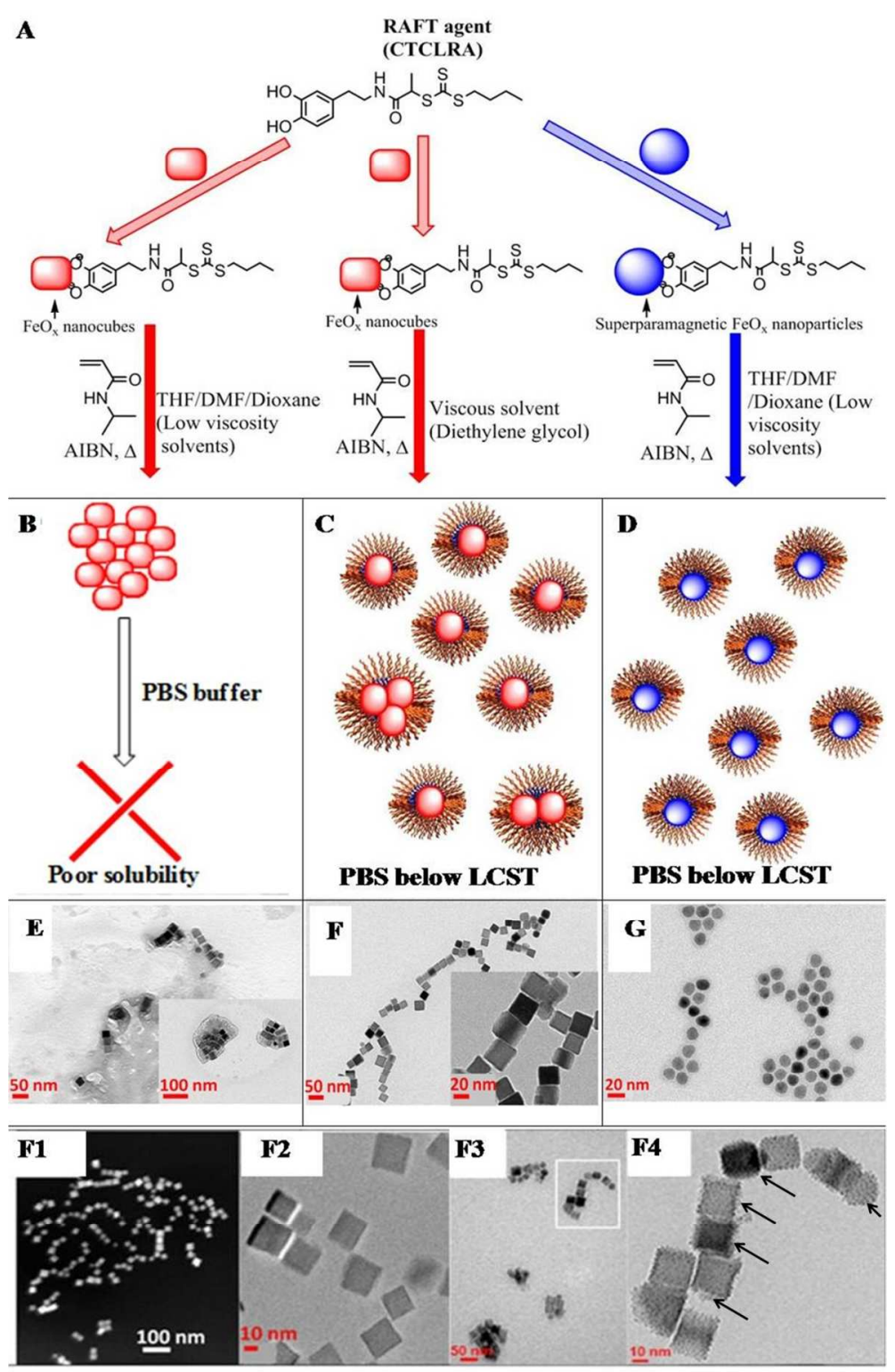

Figure 2:(A) Scheme showing the synthetic approach used to prepare thermo-responsive IONPs, (B-D) representation of the nanohybrids upon dispersion in aqueous solution, (E) TEM image of thermoresponsive cubic-IONPs obtained in THF under continuous sonication (scale bar $50 \mathrm{~nm}$ ), (F) of thermoresponsive cubic-IONPs obtained in viscous solvent (diethylene glycol) (scale bar $50 \mathrm{~nm}$ ) and (G) of 
thermo-responsive spherical-IONPs (scale bar $20 \mathrm{~nm}$ ). (F1-F4) cryo-EM images of cubic-IONPs obtained from polymerizations done in diethylene glycol (DEG). (F1) cryo-STEM image showing the chain-like assembly of nanocubes in the vitrified solution (scale bar $100 \mathrm{~nm}$ ), (F2) cryo-TEM image showing single nanocubes suspended in the vitreous solution (scale bar $10 \mathrm{~nm}$ ), (F3) cryo-TEM image showing nanocubes spontaneously arranged in chain-like assembly and small clusters (scale bar $10 \mathrm{~nm}$ ). (F4) detail of (F3) boxed region (scale bar $10 \mathrm{~nm}$ ). Note that most nanocubes are singly coated by a thin and wrinkled polymer layer (arrows) ca. $1.5 \mathrm{~nm}$ thick.

This was corroborated by dynamic light scattering (DLS) analysis of the nanocubes which showed multimodal distributions including particles with sizes close to one micron (SI, FigureS2). Furthermore, the possibility of polymer chain scission during ultrasonication for such long periods also exists. ${ }^{28-29}$ Nevertheless, the use of the ultrasonication bath presented the disadvantage of poor temperature control which led to poor reproducibility of results. To overcome these challenges, we envisioned that, in a "frozen" system, the nanocubes mobility could be reduced and hence their chance to aggregate. As a consequence, the nanocubes would stay longer in solution and polymerization could have higher chance to occur at the surface of individual nanocubes. We thus dispersed the CTCLRA functionalised cubic-IONPs in different solvents having different viscosity (either polyethylene glycol with an average molecular weight of $200 \mathrm{~g} / \mathrm{mol}$ (PEG200), or PEG400 or diethylene glycol (DEG), or THF, or dimethylformamide (DMF) or 1,4-dioxane). We placed these solutions in a heated water-bath at $60{ }^{\circ} \mathrm{C}$ to determine if the nanocubes would remain in solution or they would just precipitate as observed during the polymerization. We observed that the nanocubes in THF, DMF and 1,4-dioxane precipitated out of solution in less than half an hour whilst they remained dispersed after 6 or 24 hours in the more viscous solvents (PEG200, or PEG400 orDEG). Viscous solvents act by impairing the movement of the cubicIONPs and indeed will allow sufficient time for polymer functionalization. Moreover, such solvents have been advocated to be optimal media LRPs. ${ }^{30-31}$

Encouraged by this observation, we first studied the RAFT (co)polymerizations of NIPAAM and $\mathrm{N}$ isopropyl acrylamide mixed with polyethylene glycolmethylether acrylate (NIPAAM/PEGA) in DEG and in 1,4-dioxane, mediated by the succinimide bearing RAFT agent(SUCRA). In this experiment no nanoparticles were present. DEG was found to offer good solubility for the polymerization reagents and also its lower viscosity (compared to PEG400 and PEG200) would ensure delayed plateauing of the molar mass $\left(M_{n}\right)$. The polymerization of NIPAAM in either solvent proceeded in a controlled manner as 
evidenced by the linear increase in molar mass with conversion as well as the low polydispersity indices (PDIs) (1.1-1.3) (Figure 3). Similarly, the molar masses for the co-polymerizations of NIPAAM and PEGA were observed to increase with time and the obtained polymers also had low PD/s although the $M_{\mathrm{n}}$ versus conversion plot could not be obtained due to peak overlap in the NMR spectra. Both the NIPAAM and NIPAAM/PEGA (co)polymerizations were observed to proceed faster in the viscous solvent than those in 1,4-dioxane which is in accordance with the results published by Perrier and co-workers on LRPs in viscous solvents. ${ }^{30, b}$

Having now set a new solvent of the reaction, we then tried to perform the ligand exchange procedure with CTCLRA in the viscous solvent, by first dispersing the as-synthesized decanoic acid capped cubicIONPs in viscous solvent under ultrasonication prior to adding the CTCLRA in excess (30 molecules $/ \mathrm{nm}^{2}$ ). Success of the ligand exchange process was confirmed by FTIR as in the previous case and also by TGA (Figures S3 and S4 of the SI). We however observed no difference in surface coverage obtained using the different methods as by sulfur/iron content by ICP the final coverage ratio was found to be 4 molecules $/ \mathrm{nm}^{2}$ (see experimental section for the formula used). This suggests that the ligand exchange process was not severely impacted on by the strong interaction of the cubic IONPs. This can be explained by taking into account that in both solvent mixtures ( $\mathrm{THF} / \mathrm{CHCl}_{3}$ or dioxane or $\mathrm{DEG}$ ) there is an ultrasonication step which allows the nanocubes to become well dispersed exposing most of their surfaces for ligand exchange to take place and if the exchange process is occurring at similar rates in both cases, the surface coverage will therefore be more or less the same. Moreover, during ultrasonication heat is produced and at higher temperature the nanocubes have low tendency to interact with each other as the magnetic remanence is decreased or cancelled. After a cleaning step, functionalization of CTCLRA-cubic-IONPs (19 nm) with NIPAAM was undertaken in DEG in presence of free SUCRA to control the polymerization (see materials and methods). After removal of free polymer from the reaction mixture, the polymer coated cubic-IONPs were observed to be soluble in water or phosphate buffered saline (PBS) ( $\mathrm{pH} 7.4)$ at room temperature. 

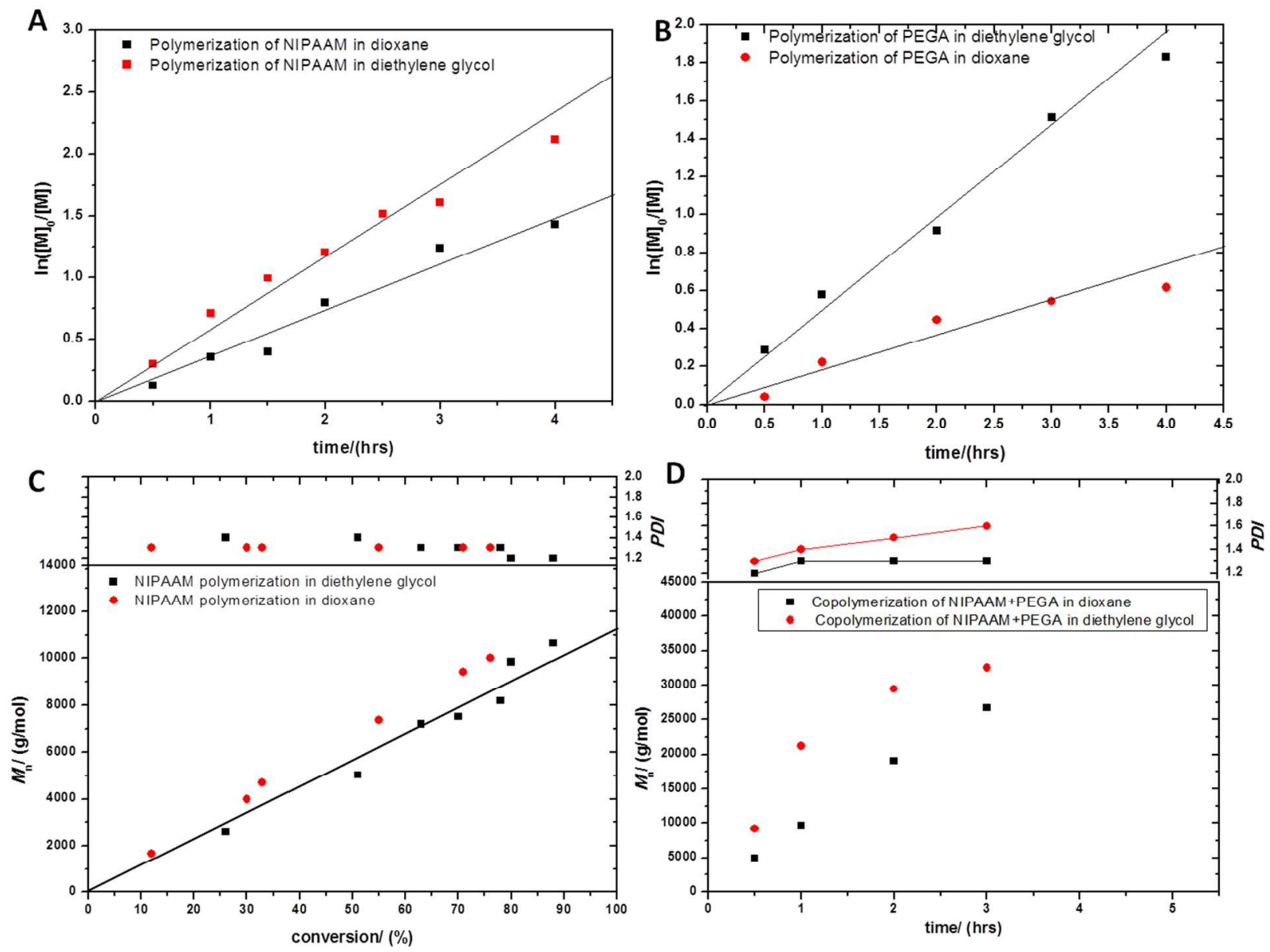

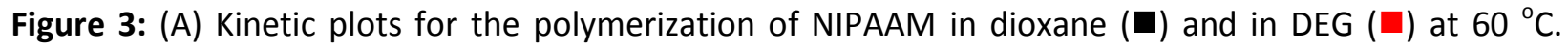
[NIPAAM]:[SUCRA]: [AIBN] was 100:1:0.2. (B) Kinetic plots for the polymerization of PEGA in dioxane

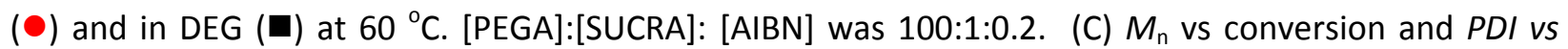
conversion graphs for the polymerization of NIPAAM in dioxane (๑) and in DEG (ם) at $60{ }^{\circ} \mathrm{C}$ [NIPAAM]:[SUCRA]: [AIBN] was 100:1:0.2. The straight line represents the theoretical $M_{\mathrm{n}}$.(D) $M_{\mathrm{n}}$ vs time and PDI vs time plot for the copolymerization of NIPAAM and PEGA in dioxane (घ) and in DEG (๑) at 60 ${ }^{\circ}$ C. [NIPAAM]:[PEGA]: [SUCRA]: ]:[AIBN] was 100:100:1:0.2.

The thermo-responsive nanocubes obtained using this procedure have a typical diameter of $c a .200 \mathrm{~nm}$ by DLS in size and showed no sign of large clusters formation as evidenced from TEM/cryo-TEM (Figure 2, F1-F4). Moreover, under cryo-TEM (Figure 2, F1-F4), it is clearly evident the quality of the monodispersed samples and the spontaneous formation of chain-like assembly of the nanocubes in the hydrated solution which might explain the high DLS values measured. Based on cryo-TEM images however, the presence of few grouping of nanoparticles as the one shown in Figure 2, F4 (see SI, Figure 
S5) were also observed suggesting the coexistence of a mixture of nanoparticles within the solution with a large proportion of single nanoparticles and some small aggregated particles.

Turbidimetric analysis confirmed the thermo-responsiveness of these hybrid nanoparticles and the LCST was ca. $29{ }^{\circ} \mathrm{C}$ which is in accordance with literature values (Figure 4). Indeed, when the nanoparticle solutions were heated above $29{ }^{\circ} \mathrm{C}$, precipitation of the particles was observed (polymer shell underwent a coil to globule transition and thus nanoparticles collapsed into macroscopical aggregates) while upon cooling below $29^{\circ} \mathrm{C}$, their solubilization occurred as the polymer became again water soluble leading to hydration of the polymer shells (Figure 4).
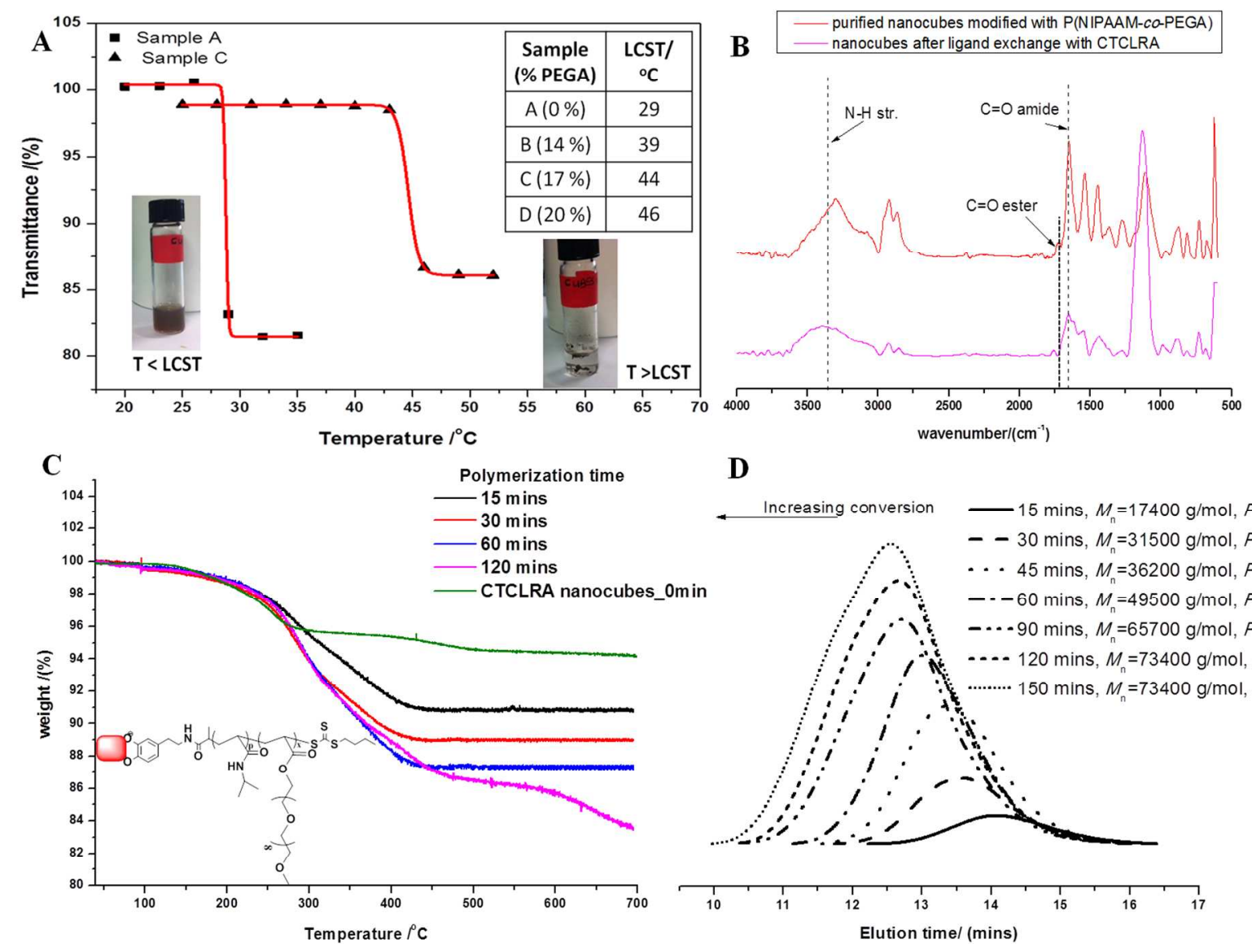

\section{D}

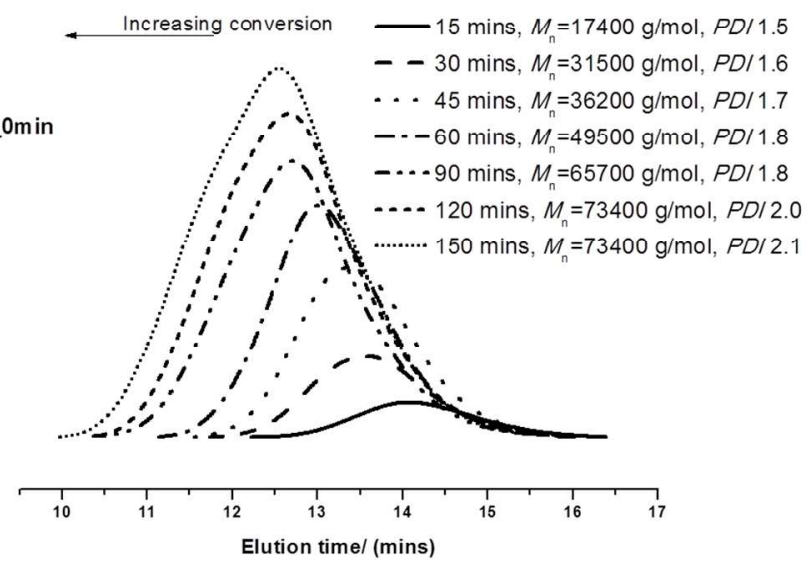

Figure 4: (A) Turbidimetric analysis of PNIPAAM (sample A) and PNIPAAM-co-PEGA functionalized cubicIONPs(sample C). The inserted table shows the variation of LCST with change in copolymerization feed composition of NIPAAM and PEGA obtained for the nanocubes. The inserted picture shows the thermoresponsive behavior of the polymer functionalized nanocubes. The vial on the left below the LCST of 
PNIPAAM (inset) shows a brown-black solution of well dispersed nanooparticles whilst the vial on the right (inset) above the LCST shows an almost colourless solution with most of the nanoparticles floating in macroscopic aggregates on top of the water and at the bottom of the glass vial. (B) FTIR spectra of iron oxide nanocubes functionalised with CTCLRA before and after polymerization with PNIPAAM/PEGA in diethylene glycol. (C) Thermogravimetric analysis of PNIPAAM-co-PEGA functionalized cubic-IONPs (structure in inset) obtained at different polymerization times and (D) SEC chromatograms obtained by analysis of free polymers during the functionalization of iron oxide nanocubes via RAFT polymerization at different times.

As we sought to prepare thermo-responsive IONPs for cancer treatment with thermal response significant for hyperthermia $\left(41-47^{\circ} \mathrm{C}\right),{ }^{15,32}$ we targeted coil-globule response temperatures above body temperature $\left(37^{\circ} \mathrm{C}\right)$ and maintaining this behavior in physiological conditions (saline buffer solutions at $155 \mathrm{mM}$ of $\mathrm{NaCl}$ ). Copolymerization of NIPAAM with hydrophilic monomers increases the LCST and thus, we chose to copolymerize NIPAAM with polyethylene glycol methylether acrylate (PEGA). In addition to offering hydrophilicity, polyethylene glycol also has the advantages of being non-toxic $\left(M_{n}>400\right.$ Da but below $40 \mathrm{kDa}$ ) as well as reducing in vivo protein adsorption and thus improving biodistribution although few drawbacks PEG dose and PEG molecular weight related should not be neglected (i.e. immunogenic issues due to complement activation and antibody response). ${ }^{33}$ Varying in the feed, the initial mole percentage of hydrophilic monomer (PEGA) between 0 and $35 \%$ with respect to that of NIPAAM we were able to tune the LCST to a temperature above $37^{\circ} \mathrm{C}$ thus ensuring cargo (drug) encapsulation and water solubility at temperatures below this value and conversely cargo release at temperatures recommended for hyperthermia above $40{ }^{\circ} \mathrm{C}$ (Figure 4).

Thus, using our functionalization procedure, the LCST of the hybrid cubic-IONPs could be easily modified by copolymerizing NIPAAM with PEGA (Figure 4A). Below LCST the nanocubes were completely soluble while above the LCST, the nanocubes collapsed (inset images of figure $4 \mathrm{~A}$ ) and this behavior was totally reversible by switching the temperature. The copolymerization with a monomer feed composition of $80 \%$ mol NIPAAM and $20 \%$ mol PEGA was studied over time and found to give hybrid nanostructures with an LCST of $45^{\circ} \mathrm{C}$. Analysis of the thermo-responsive cubic-IONPs by TGA revealed an increase in \% weight loss with increasing polymerization time which corresponds with gradual molar mass increase observed in LRPs (Figure 4C). Analysis of the free polymers by size exclusion chromatography (SEC) indicated that the molar mass increased with time although the PDI values were relatively high (1.5-1.7) (Figure 4D). These $P D I$ values were however lower than those obtained when the polymerizations were 
conducted using oleic acid capped cubic-IONPs in the absence of free RAFT agent (2.5-4.3) suggesting the grafting from polymerizations done using CTCLRA coated nanocubes was controlled albeit with less efficiency. Finally, by FTIR, the amide $(\mathrm{C}=\mathrm{O}$ and $\mathrm{N}-\mathrm{H})$ and ester $(\mathrm{C}=\mathrm{O})$ peaks indicate the presence of the thermo-responsive polymer shell present at the nanocube surface (Figure 4B).

The thermo-responsive cubic-IONPs also exhibited magnetization properties (at low temperature, 5K) similar to those of the as synthesized or PEG water transferred cubic-IONPs reported previously (Figure 5). ${ }^{22}$ Noteworthy, at $300 \mathrm{~K}$ and high magnetic fields (above $5 \mathrm{kOe}$ ) the magnetization signal for the polymer coated nanocubes system decreases while increasing the magnetic field (Figure 5B). This is due to the diamagnetic contribution of the polymer which becomes relevant at high temperatures and fields. ${ }^{34}$ As a result, and depending on the amount of polymer (and possible solvent entrapped on/in it) a negative susceptibility at high magnetic fields and at high temperatures is observed. For the thermoresponsive nanocubes, this further corroborates the presence of a polymer, the thermo-responsive shell, at the surface of nanocubes.
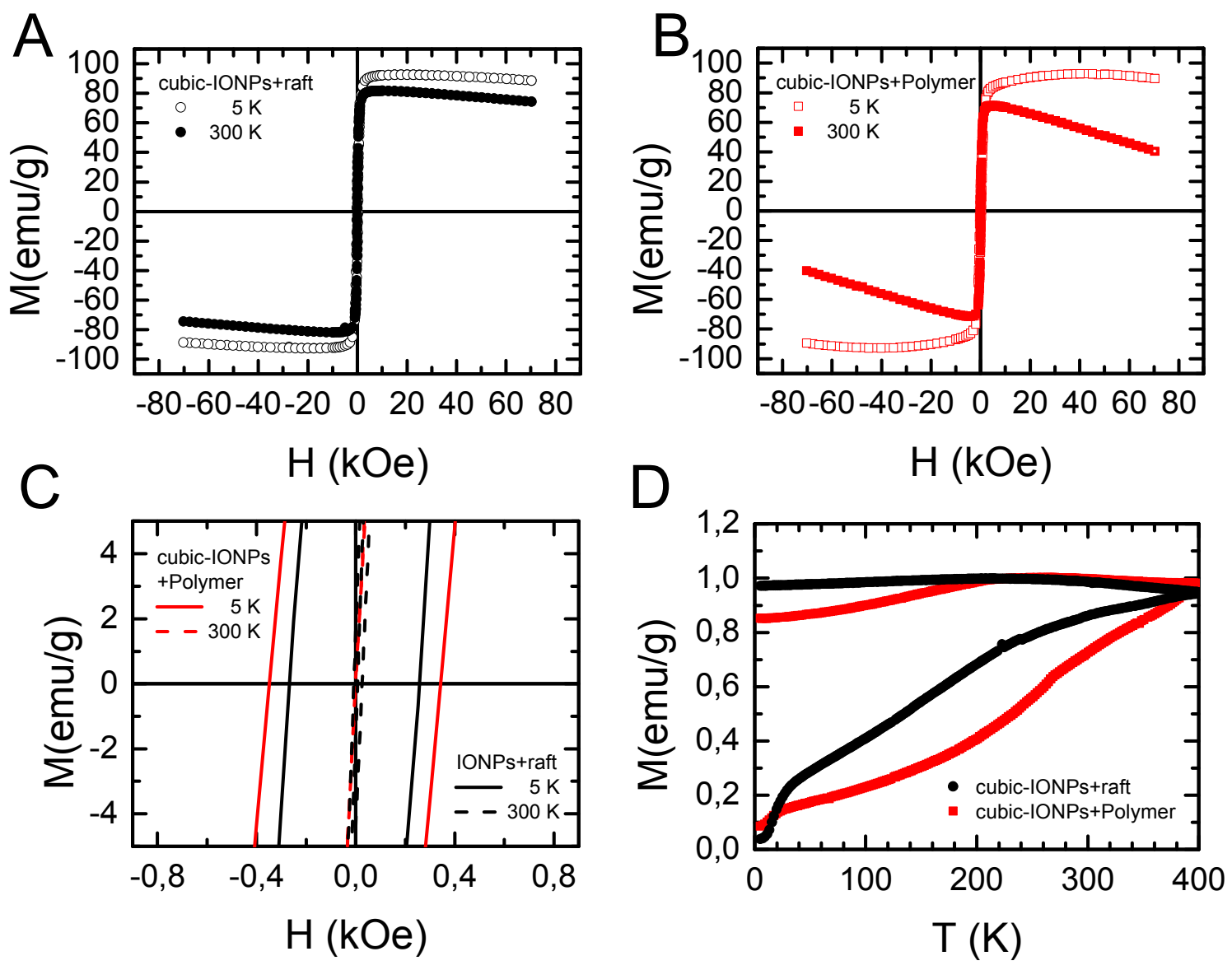
Figure 5: (A) Hysteresis loops at $5 \mathrm{~K}$ (open black circles $\mathrm{O}$ ) and $300 \mathrm{~K}$ (black circles 9 ) for $19 \mathrm{~nm}$ iron oxide nanocubes- functionalized with CTCRLA, the raft agent and (B) the same nanoparticles after having grown the thermo-responsive polymer $5 \mathrm{~K}$ (open red square $\square$ ) and $300 \mathrm{~K}$ (red square $\square$ ). Notice how the diamagnetic signal contribution derived from the polymer increases while increasing temperature. (C) Low field detail of the magnetization hysteresis loops at $5 \mathrm{~K}$ (solid line) and $300 \mathrm{~K}$ (dashed line) for CTCRLA raft functionalized (black line) and thermo-responsive polymer coated (red line) $19 \mathrm{~nm}$ iron oxide nanocubes. (D) Temperature dependence of the zero field cooling and field cooling (cooling field $\mathrm{H}_{\mathrm{FC}}=100 \mathrm{Oe}$ ) magnetization curves measured at $100 \mathrm{Oe}$, as a function of particle size for CTCRLA raft functionalized (black circles $\bullet$ ) and thermo-responsive polymer coated (red square $\square$ ) $19 \mathrm{~nm}$ iron oxide nanocubes. For both samples the blocking temperature $\left(T_{B}\right)$ is above room temperature.

\subsection{Cytotoxicty assay}

Besides tuning the phase transition temperature for feasible applications at body temperature, for any material to be applicable as a drug delivery vehicle, it is imperative that the material is non-cytotoxic. We studied the effect of our polymer coated (PNIPAAM-Co-PEGA) nanocubes on Kb cells by conducting cell viability and cell uptake studies. Cell viability studies showed no difference in $\%$ viability between $\mathrm{Kb}$ cells incubated for 16 hours with polymer coated nanocubes at high concentration ( $1 \mathrm{~g} / \mathrm{L}$ of iron) and $\mathrm{Kb}$ cells treated only with the corresponding amount of PBS, used as control; in a parallel experiment under the same conditions, cells that were rinsed from the excess of IONP and kept for additional 32 hours in incubation, also showed very high viability(in this case the total exposure time of cells to the cubicIONPs is of $48 \mathrm{~h}$ ), indicating the nanohybrids were not cytotoxic (Figure 6A). On the other hand, as confirmed by measuring the iron amount by ICP, the uptake of the thermo-responsive coated nanocubes is slightly higher than that of similar nanocubes coated by only PEG or enwrapped in an amphiphilic polymer and as previously found by $\mathrm{us}^{22}$ (250 picomol/cells versus 200 and 150 respectively) which does corresponds roughly to $20 \%$ of the administered dose (Figure 6B). It is important to note that the high amount of magnetic material incubated for 16 hours in the cell dish formed a dark layer on top of the adherent cells. This was clearly visible under optical phase contrast microscopy on cell plate (after having rinsed the excess of nanoparticle in solution), and is due to the high level of IONPS internalized by the cells or deposited at the cell surface (Figure 6B, right upper panel). However despite the high amount of iron associated to the cells, the Kb cells kept their normal morphology and the nanocubes did not appear to be toxic (Figure 6B, upper panel). Worthily, despite the very high 
concentration of nanoparticles administered, the stability of the IONPs in cell medium was not compromised, leading to very few aggregates that do not cause any toxic response (figure S11 in the ESI).
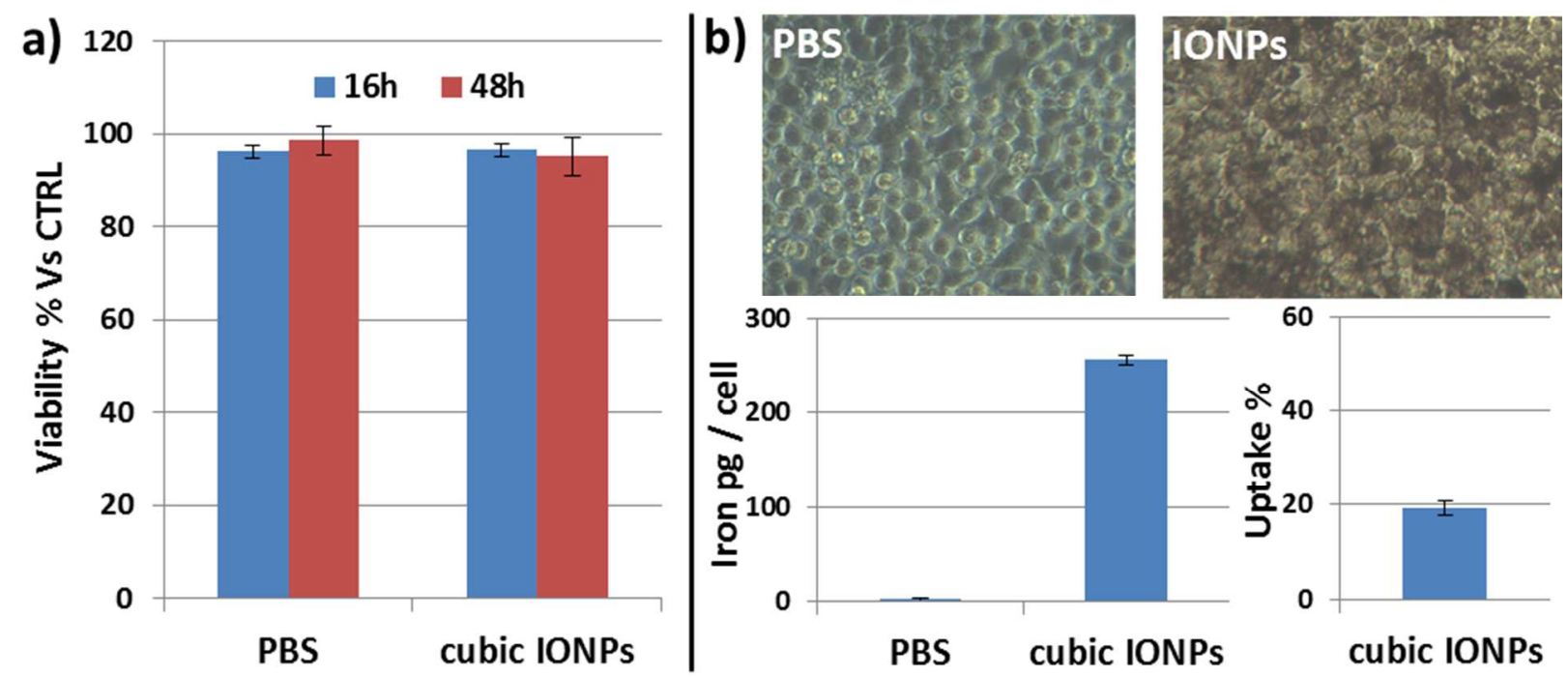

Figure 6: Cell study on Kb cells: (A) \% of viability assessed by trypan blue assay after 16 and $48 \mathrm{~h}$ of incubation with thermo-responsive cubic-IONPs ( $1 \mathrm{~g} / \mathrm{L}$ of iron) with respect to a cell control sample (CTRL) to which a corresponding volume of PBS (no nanoparticles) was administered; (B) Uptake analysis: the phase contrast images above show how the cells appear before (left) and after (right) $16 \mathrm{~h}$ of incubation with thermos-responsive-cubic-IONPs ( $1 \mathrm{~g} / \mathrm{L}$ of iron); the panels below show the amount of iron per cell measured by ICP elemental analysis for two cell samples treated under the same conditions but one exposed to the IONPs and the other to just buffer (left), and the percentage of iron uptake with respect to the extracellular iron (1300 pg iron per cell) added to the cell media (right).

\subsection{Drug loading and release experiments}

Cubic-IONPs (LCST of sample, $47^{\circ} \mathrm{C}$ ) were loaded with doxo at room temperature and in order to demonstrate the temperature dependence of doxo release, the drug release was assessed at $4{ }^{\circ} \mathrm{C}, 25^{\circ} \mathrm{C}$, $37{ }^{\circ} \mathrm{C}$ and at $50{ }^{\circ} \mathrm{C}$ (above LCST of copolymer). For the loading, thermo-responsive coated cubic-IONPs (50 ppm Fe) were mixed with doxo $(150 \mu \mathrm{g}$ in $2 \mathrm{~mL}$ ) and after a 24 hour incubation period, the excess (free) doxo was removed by at least five washing steps in which the drug loaded nanocubes were 
collected by means of a magnet leaving in solution the excess of doxo which was measured and then discarded. The drug loading achieved was $44.75 \%$ with respect to the initial doxo amount, , as measured by difference between the initial doxo absorbance and the absorbance of the supernatants (which contains the doxo not loaded into the polymer shell) found in the 5 washing steps. This also means that $67.125 \mu \mathrm{g}$ of doxo were associated per $0.1 \mathrm{mg}$ of iron. The release of the drug from the dispersion of doxo loaded nanoparticles ( $200 \mathrm{ppm}$ in Fe) was found to be only $8 \%$ at $37^{\circ} \mathrm{C}$ over 24 hours and even less at $25{ }^{\circ} \mathrm{C}$ and $4{ }^{\circ} \mathrm{C}$. However, more than $90 \%$ of the drug was released in less than 5 hours at $50{ }^{\circ} \mathrm{C}$ clearly suggesting that the release was heat triggered (Figure 7A). In these cases a water bath was used to increase the temperature and after thermal treatment the thermo-responsive-IONPs were collected to the magnet and the supernatant analyzed for the doxo quantification by absorption. We also extended our study to demonstrate the "on-demand" and remotely triggered drug release by conducting drug release under an external activation, the AMF. We therefore prepared another sample (LCST, $\left.52{ }^{\circ} \mathrm{C}\right)$ of drug loaded cubic-IONPs as described above but on a larger scale (6 mg Fe diluted to $50 \mathrm{ppm}$ ) and the drug loading achieved in this case was $23 \%$ (2.2 mg of doxo). Doxo release was observed to take place from drug loaded thermo-responsive cubic-IONPs when the concentration of nanocubes in the solution was fixed at $3.8 \mathrm{~g} / \mathrm{L}$ (of iron) and under an AMF $\left(220 \mathrm{kHz}, 20 \mathrm{kAm}^{-1}\right)$ whilst negligible release was observed for a similar sample not subjected to an AMF (Figure 7B and SI, Figure S6) and kept at $25{ }^{\circ} \mathrm{C}$. About $25 \%$ of the loaded drug was released over 240 minutes (Table S1)."

During the AMF exposure at this nanocube concentration, the temperature of the solution reached 80 ${ }^{\circ} \mathrm{C}$ in the first 15 minutes which is clearly above the LCST of $52{ }^{\circ} \mathrm{C}$ of the thermo-responsive sample employed. Remarkably, the temperature profile of the PNIPAAM-co-PEGA cubic-IONPs is comparable to that of the same batch of cubic-IONPs brought in water by a chatecol-PEG using a ligand exchange procedure developed by our group (Figure 7C). ${ }^{22}$ It is worth mentioning that when we used the waterbath, we could achieve the release even at nanocube concentration as low as $0.2 \mathrm{~g} / \mathrm{L}$ of iron. 

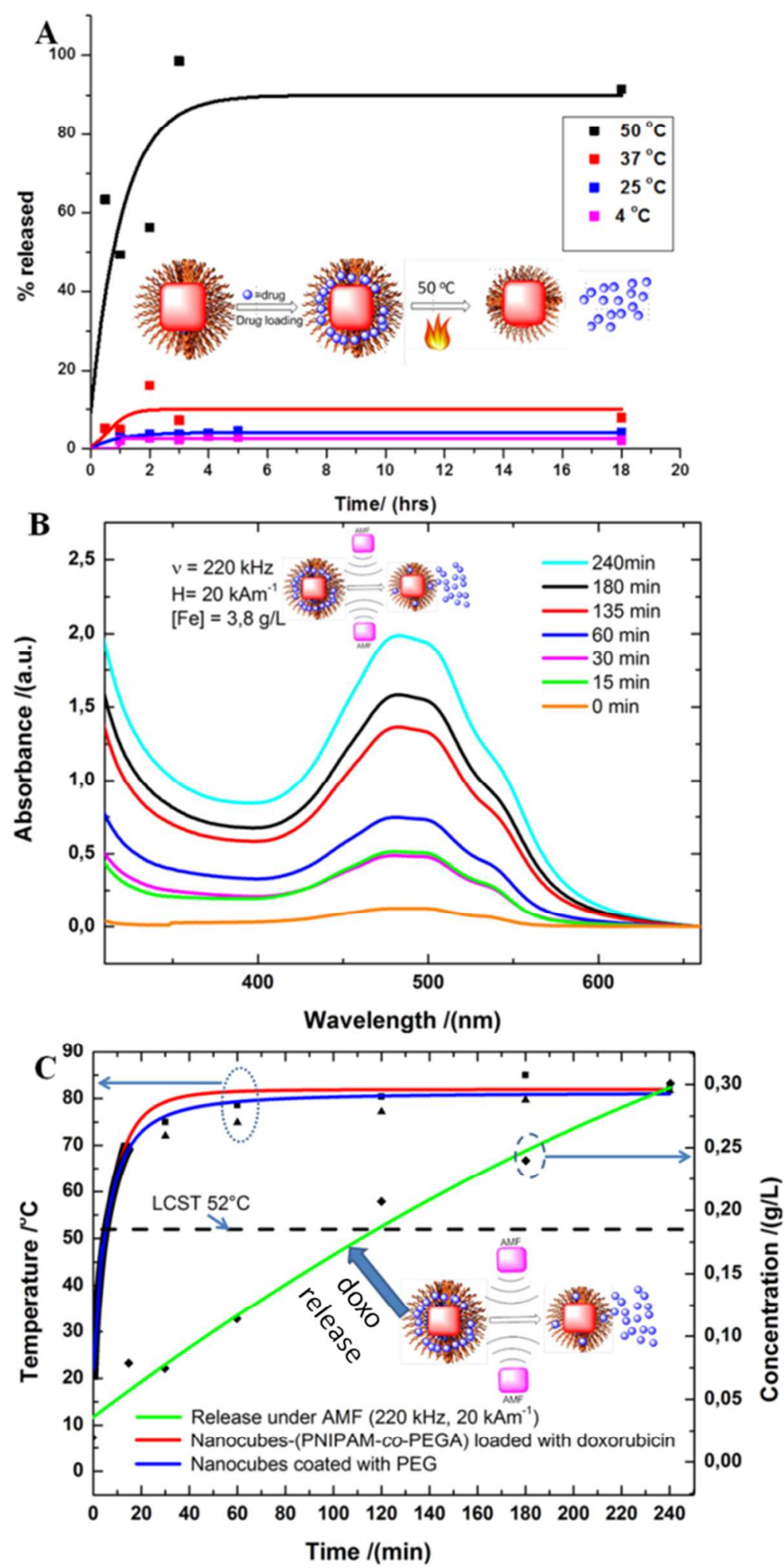

Figure 7: (A) Drug release from thermo-responsive PNIPAAM-co-PEGA cubic-IONPs in a water bath at various temperatures $\left(50{ }^{\circ} \mathrm{C} \mathbf{\square}, 37{ }^{\circ} \mathrm{C} \square, 25{ }^{\circ} \mathrm{C} \square\right.$ and $\left.4{ }^{\circ} \mathrm{C} \square\right)$, (B) Absorption spectra obtained at 
different times showing release of doxo under AMF from PNIPAAM-Co-PEGA cubic-IONPs and (C) Comparison of the temperature profiles of PNIPAAM-Co-PEGA functionalized cubic-IONPs (red line, ( $\mathbf{\square})$ ) and PEG functionalized cubic-IONPs (blue line, $(\boldsymbol{\bullet})$ ) at $3.8 \mathrm{~g} / \mathrm{L}$ [Fe](left axis) and the concentration of doxo release (right axis, green line $(\bullet)$ ) over time.

However, under AMF, the temperature of a $0.2 \mathrm{~g} / \mathrm{L}$ of iron solution did not increase and remained constant at $25{ }^{\circ} \mathrm{C}$ and under those conditions no drug release was observed. This suggests that the polymer shrinking with consequent drug release is a collective property which involves inter-particle interaction rather than a single particle shrinking process. In other words, to activate the shrinking of the nanosystem, a macroscopical temperature increase rather than local heating effects plays a major role.

\subsection{Implementation of the polymerization procedure on nanocubes with $\mathrm{pH}$-responsive monomer units}

As a further extension of this work, to demonstrate the versatility of our synthetic approach, we also prepared nanocubes functionalized with a pH-responsive polymer shell, poly(vinyl pyridine) (PVP) by simply choosing a different monomer, 4-vinylpyridine (VP) and keeping all the other conditions as employed for the thermo-responsive shell polymerization. The $\mathrm{pH}$-responsive polymer functionalized nanocubes were water soluble below $\mathrm{pH} 5$ due to protonation of the pyridine groups of the polymer (positive zeta potential) while they precipitated out of water above $\mathrm{pH} 5$ due to neutralization of the positive charge (zeta potential $\leq 0$ ) (Figure 8). Copolymerization of VP $(50 \%)$ with PEGA (50\%) gave pHresponsive cubic-IONPs that were water soluble even above $\mathrm{pH} 5$ indicating the water solubilizing effect of PEG (ESI, Tables S2 and S3, FigureS7). 


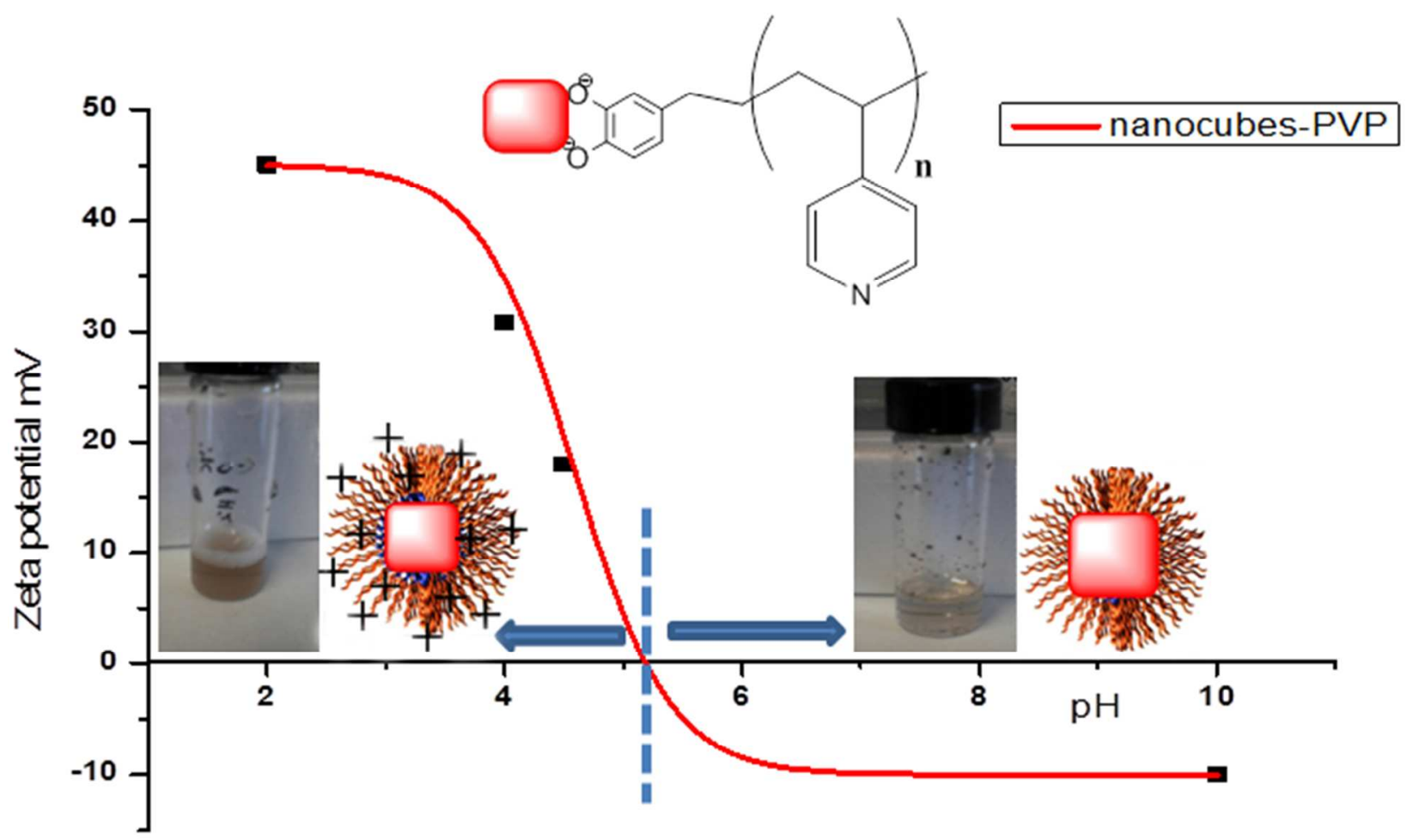

Figure 8: Zeta potential as a function of $\mathrm{pH}$ of PVP functionalized cubic-IONPs. The protonation of PVP below $\mathrm{pH} 5$ allows the stabilization of the nanocube in solution by electrostatic repulsions. The vial on the left below the $\mathrm{pK}_{\mathrm{a}}$ of PVP (inset) shows a brown solution of well dispersed nanooparticles whilst the vial on the right (inset) above the $\mathrm{pK}_{\mathrm{a}}$ shows an almost colourless solution with most of the nanoparticles stuck on the wall of the glass vial.

\subsection{Control polymerization experiments using superparamagnetic and spherical IONPs}

To confirm that the complication in functionalization of the cubic-IONPs resulted from their strongly interacting character, we functionalized spherical superparamagnetic IONPs synthesized by following the protocol reported by Colvin and coworkers. ${ }^{35}$ Due to their superparamagnetic behavior at room temperature, these spherical-IONPs have no tendency to aggregate. The oleic acid capped sphericalIONPs were easily exchanged with the CTCLRA (see materials and method section) and on the CTCLRA functionalized nanoparticles polymerization with NIPAAMor PNIPAAM-Co-PEGA could be undertaken with no difference either in dioxane or in THF affording water or PBS buffer ( $\mathrm{pH}$ 7.4) dispersible thermoresponsive spherical-IONPs. In this case, no precipitation or aggregation was observed over entire 
duration of the polymerization which is similar to the observations made in the work by Onho et al. ${ }^{26}$ in which non-aggregating superparamagnetic IONPs were successfully functionalized via surface mediated RAFT polymerization. Analysis of the typical coated nanoparticles obtained by TEM also showed a thin shell of polymer around the nanoparticles and the complete absence of clusters (Figure 2G). In addition, an increase in size after polymerization was observed by DLS and thermo-responsiveness of the nanohybrids was ascertained by turbidimetric analysis (SI, Figure S9-S10). This direct comparison just confirms that cubic-IONPs, which are strongly interacting nanoparticles, are more challenging to functionalize with respect to superparamagnetic IONPs which do not interact at all with each other.

\section{Conclusion}

In summary, we have developed a facile procedure to functionalize strongly interacting magnetic nanoparticles via RAFT polymerization with stimuli-responsive polymers and generate smart nanomaterials that have potential application in the biomedical field. Using our procedure, we successfully functionalized iron oxide nanocubes with a thermo-responsive and also with a $\mathrm{pH}$ responsive polymer to obtain responsive biocompatible nanohybrids. The exploitation of the thermoresponsive hybrids was demonstrated by loading and release of an anti-cancer drug (doxo) into the nanohybrids which could be release, on demand, under an AMF due to the heat generated by the nanocubes. Such materials may provide a synergistic therapeutic effect in cancer treatment via hyperthermia and chemotherapy with triggered drug release while not displaying the side effects of standard chemotherapy.

\section{Experimental Section}

4.1 Materials. N-isopropylacrylamide (NIPAAM) (Aldrich, 99\%) and $\mathrm{N}$-isopropylmethacrylamide (NIPMAM) (Aldrich, 99\%) were purified by re-crystallization from hexane three times and dried under vacuum at room temperature before use. Poly(ethylene glycol) methyl ether acrylate (average $M_{n} 480$ ) (Aldrich) and 4-vinyl pyridine (Aldrich) were destabilized by passing the monomer through a short column packed with inhibitor remover (Aldrich). Azobis(isobutyronitrile) (AIBN) was purified by recrystallization from methanol twice and then it was left to dry under reduced pressure in a desiccator. 1,4 Dioxane (Sigma Aldrich, 99\%) and tetrahydrofuran (THF) were distilled and stored under nitrogen 
prior to use. The trithiocarbonate RAFT agent, 2-(butylthiocarbonothioylthio) propanoic acid (BTTPA) was synthesized in accordance with a previously published protocol. ${ }^{36}$ Doxorubicin hydrochloride and all other solvents were purchased from Sigma Aldrich at the highest purity available and used as received. Tetra-N-butylammonium bromide was obtained from Merck and used as received. Cubic or spherical iron oxide nanoparticles, (cubic-IONPs and spherical-IONPs) were prepared in accordance with previously reported methods. ${ }^{21,37,22}$

\subsection{Synthesis of succinimide RAFT agent (SUCRA).}

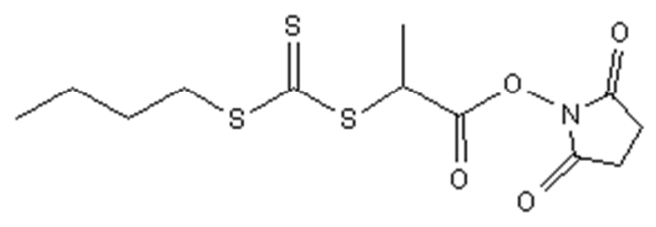

Scheme 1: SUCRA.

To a $500 \mathrm{~mL}$ round bottom flask was added BTTPA (10.00 g, $42.00 \mathrm{mmol}), \mathrm{N}$-hydroxysuccinimide (7.25 g, $63.02 \mathrm{mmol}), \operatorname{DMAP}(0.51 \mathrm{~g}, 4.20 \mathrm{mmol})$ and dry THF $(200 \mathrm{~mL})$. The reaction vessel was placed in an ice bath and the contents were stirred for 15 minutes followed by dropwise addition of DIC $(10.60 \mathrm{~g}, 84.00$ $\mathrm{mmol})$ in $\operatorname{THF}(50 \mathrm{~mL})$. The reaction was kept at $0{ }^{\circ} \mathrm{C}$ for 4 hours before it was allowed to gradually warm up to room temperature and then left to stir for 24 hours. At the end of the reaction, the reaction mixture was filtered via gravitational filtration to remove a white precipitate. Solvent from the collected filtrate was removed in vacuo to yield a yellow residue which was taken up in dichloromethane and transferred to a separating funnel. The organic layer was washed with water $(3 x)$ and brine $(1 x)$ then dried over $\mathrm{MgSO}_{4}$. After filtration to remove $\mathrm{MgSO}_{4}$, removal of solvent in vacuo afforded the crude product which was purified via flash chromatography $\left(\mathrm{SiO}_{2}\right.$, Initially, Hexane 95\%: Ethyl acetate 5\% for approximately 5-6 column volumes (elutes yellow coloured impurities, product does not move) then Hexane 65\%: Ethyl acetate $35 \%$ (elutes product, $R_{f} 0.65$ ). Removal of solvent in vacuo gave the product as a viscous yellow oil $(12.30 \mathrm{~g}, 87 \%)$. For the ${ }^{1} \mathrm{H}$ NMR characterization see Figure $\mathrm{S} 1$ (SI) ( 0.93 (t, $J=7.3$ $\mathrm{Hz}, 3 \mathrm{H}$ ), 1.43 (sextet, J=7.5 Hz, 2H), 1.75 (d, J=7.4 Hz, 3H), $1.69(\mathrm{~m}, 2 \mathrm{H}), 2.83(\mathrm{~s}, 4 \mathrm{H}), 3.38$ (t, J=7.4 Hz, $2 \mathrm{H}), 5.15(\mathrm{q}, J=7.4 \mathrm{~Hz}, 1 \mathrm{H})$

\subsection{Synthesis of catechol functionalised RAFT agent (CTCLRA).}




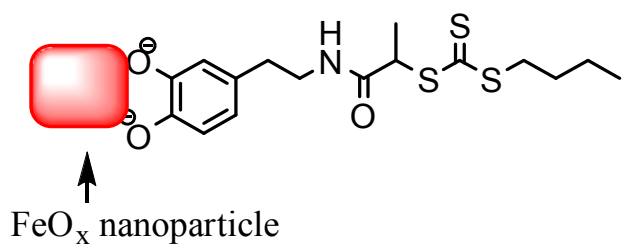

Scheme 3: Cubic-IONPs functionalized with CTCLRA.

The stock solution of iron oxide nanocubes (see below) was placed in the sonication bath for fifteen minutes after which the desired volume (see below) was transferred to a glass vial followed by ultrasonication at $0{ }^{\circ} \mathrm{C}$ in an ice bath for one minute. To a solution of CTCLRA ( $7 \mathrm{mmol}$ in $50 \mathrm{~mL}$ THF) was added to the nanocubes ( $5 \mathrm{~mL}$ in chloroform, $0.14 \mu \mathrm{M}$, [Fe] $2.0 \mathrm{mg} / \mathrm{mL}, 18 \pm 2 \mathrm{~nm}$ edge length as by TEM) and then triethylamine ( $1 \mathrm{~mL}$ in $50 \mathrm{mLTHF}$ ) and the resulting colloidal solution was ultrasonicated for 1 minute at $0{ }^{\circ} \mathrm{C}$ in an ice bath then transferred to a sonication bath with sonication on full power (30 W) to keep the cubes suspended in solution. After sonication for two hours, the vial was transferred to the 
shaker and left to shake vigorously overnight. The nanoparticles were recovered by centrifugation at $3500 \mathrm{rpm}$ for 10 minutes and the collected nanoparticles were re-dispersed in THF. Hexane was added to near the point of particle precipitation (the amount of hexane added was such that no particle or CTCLRA precipitation was observed but further addition of hexane, few $\mathrm{mL}$, would result in particle precipitation). The solution was centrifuged to recover the nanoparticles and the supernatant solution was decanted. This process was repeated until the characteristic yellow color of the RAFT agent in the supernatant disappeared after which the nanoparticles were washed two more times. The functionalized nanoparticles were insoluble in hexane which is a good solvent for the starting nanoparticles thus indicating the change in the coating at their surface.

ICP (starting nanoparticles), $[\mathrm{Fe}]=2 \mathrm{~g} / \mathrm{L},[\mathrm{S}]=0 \mathrm{ICP}$ (CTCLRA functionalized nanoparticles), $[\mathrm{Fe}]=1.98$ $\mathrm{g} / \mathrm{L},[\mathrm{S}]=0.1 \mathrm{~g} / \mathrm{L}$ (calculated based on same volume of starting and CTA functionalized nanoparticles).

FTIR: C=S $1060 \mathrm{~cm}^{-1}$, overlaps with nanocubes peak. $\mathrm{N}-\mathrm{H}, 3400 \mathrm{~cm}^{-1}$. Initiator grafting density (molecules $/ \mathrm{nm}^{2}$ ) was calculated using the formula $\left(C_{\text {sulfur }} / 96\right) /\left(C_{N P S} \times 6 S_{\text {TEM }}{ }^{2}\right)$. $C_{\text {sulfur }}$ is the concentration of sulfur from ICP in $\mathrm{g} / \mathrm{L}, \mathrm{S}_{\text {TEM }}$ is the average edge size of the nanoparticles from TEM in nanometers and $\mathrm{C}_{\mathrm{NPs}}$ is the concentration of nanoparticles (mol nanoparticles/L).

\subsection{Functionalization of cubic IONPs with thermo-responsive polymer (PNIPAAM) in low viscosity solvents without sonication (Example procedure).}

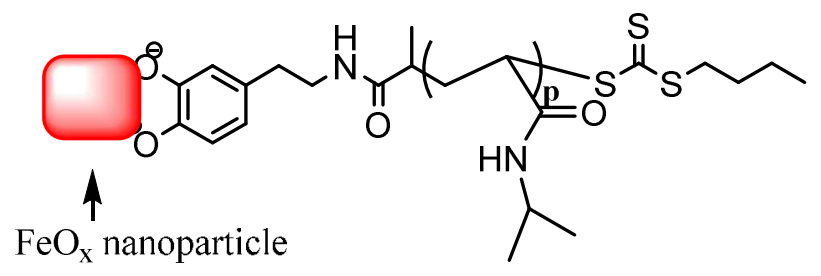

Scheme 4: Cubic-IONPs functionalized with thermo-responsive polymer (PNIPAAM).

To a glass vial was added NIPAAM (4.000 g, $35.4 \mathrm{mmol})$, SUCRA $(0.011 \mathrm{~g}, 32.8 \mu \mathrm{mol})$, solvent (dioxane or THF) ( $4 \mathrm{~g}$ ), and CTCLRA coated iron oxide nanocubes dispersed in THF ( $1 \mathrm{mg}$ Fe, from $0.6 \mu \mathrm{M}$ stock solution of NPs). The solution was vortexed for a few seconds and the vial was sealed with a rubber septum followed by degassing with nitrogen gas for 30 mins. AIBN ( $25 \mu \mathrm{L}, 3.28 \mu \mathrm{mol})$ was then added from a stock solution in dioxane via a microliter syringe and degassing was continued for a further 10 minutes. The reaction contents were mixed for a few seconds and the vial was placed in a thermostated 
water bath at $60^{\circ} \mathrm{C}$ for $3 \mathrm{~h}$. The reaction was stopped by placing the vial in an ice bath and opening it to the atmosphere. A sample for analysis by SEC was obtained by taking an aliquot of the reaction mixture (ca. $100 \mathrm{mg}$ in around $100 \mu \mathrm{L}$ of the sample) which was diluted with THF and placed on a magnet to remove the nanoparticles prior to collection of the supernatant for analysis. The remainder of the reaction mixture was precipitated into cold diethyl ether and the collected precipitate was dissolved in a small amount of THF and reprecipitated in cold diethyl ether. This process was repeated one more time and the precipitate was then dissolved in THF/diethyl ether (1:2 in volume) and centrifuged at $5000 \mathrm{rpm}$ for 10 minutes. The supernatant was decanted into cold hexane were precipitation of free polymer could be observed. The precipitated nanoparticles were resuspended in THF/diethyl ether (1:2) and centrifuged again at $5000 \mathrm{rpm}$ for 10 minutes and this approach was repeated 3 times with the supernatant being poured into cold hexane. The supernatant from the last wash was concentrated on the rotary evaporator and analysed by SEC to confirm the absence of free polymer. In this case (no continuous sonication), the cubic-IONPs precipitated during the early stages of polymerization (ca. 10 mins).

4.6 Functionalization of cubic IONPs with thermo-responsive polymers (NIPAAM) in low viscosity solvents under continuous sonication. The procedure followed is similar to that detailed above (when no sonication was employed) with the only difference being that instead of conducting the reaction in a thermostated water bath, the reaction vessel was placed in a sonication bath with sonication set at full power $(30 \mathrm{~W})$ and the temperature set at $60{ }^{\circ} \mathrm{C}$ for the entire duration of the polymerization. Worthily, in this case (under continuous sonication), the cubic-IONPs did not precipitate as observed in the previous case when no sonication was employed during the polymerization. However, though the temperature was set at $60^{\circ} \mathrm{C}$, it fluctuated significantly $\left(60-85^{\circ} \mathrm{C}\right)$ during the polymerization and it was very difficult to keep it within a small range of fluctuation $\left( \pm 2^{\circ} \mathrm{C}\right)$ leading to poor reproducibility of results.

4.7 Functionalization of iron oxide nanocubes with CTCLRA after initially dispersing the IONPs in viscous solvent (Method 2). $\mathrm{A} \mathrm{CHCl}_{3}$ solution of decanoic acid capped iron oxide nanocubes $(5 \mathrm{~mL}, 0.14$ $\mu \mathrm{M},[\mathrm{Fe}] 2.0 \mathrm{mg} / \mathrm{mL}, 18 \pm 2 \mathrm{~nm}$ edge length (TEM)) was added to a glass vial containing diethylene glycol $(20 \mathrm{~mL})$ which was sealed with a suba seal and degassed with nitrogen to evaporate off most of the $\mathrm{CHCl}_{3}$. The resulting solution was vortexed and cooled to $0{ }^{\circ} \mathrm{C}$ in an ice bath before it was subjected to ultrasonication for 20 minutes. To the resulting solution of nanocubes was added a solution of CTCLRA ( $7 \mathrm{mmol}$ in $50 \mathrm{~mL}$ THF ) and triethylamine (1 mL in $50 \mathrm{~mL}$ THF) and the colloidal solution was 
ultrasonicated for a total of 20 minutes at $0{ }^{\circ} \mathrm{C}$ in an ice bath (ultrasonication cycle, 30 seconds on , 30 seconds off) then left on a shaker overnight. The nanoparticle solution was then diluted with THF (1:3) and centrifuged at $4000 \mathrm{rpm}$ for 10 minutes to recover the nanoparticles. The collected nanoparticles were redispersed in THF $(20 \mathrm{~mL})$ before hexane was added to near the point of particle precipitation (the amount of hexane added was such that no particle or RAFT agent precipitation was observed but further addition of hexane (few $\mathrm{mL}$ ) would result in particle precipitation). The solution was centrifuged at $4000 \mathrm{rpm}$ for 10 minutes to recover the nanoparticles and the supernatant solution was decanted. This process was repeated with THF/hexane until the characteristic yellow color of the RAFT agent in the supernatant disappeared after which the nanoparticles were washed three more times. The functionalized nanoparticles were insoluble in hexane which is a good solvent for the starting nanoparticles. ICP (starting nanoparticles), $[\mathrm{Fe}]=2 \mathrm{~g} / \mathrm{L},[\mathrm{S}]=0 . \quad \mathrm{ICP}$ (CTCLRA functionalisednanoparticles) ), $[\mathrm{Fe}]=1.98 \mathrm{~g} / \mathrm{L},[\mathrm{S}]=0.1 \mathrm{~g} / \mathrm{L}$ (calculated based on same volume of starting and CTCLRA functionalized nanoparticles). FTIR: C=S $1060 \mathrm{~cm}^{-1}$, overlaps with nanocubes peak. $\mathrm{N}-\mathrm{H}, 3400 \mathrm{~cm}^{-1}$. Initiator grafting density (molecules $/ \mathrm{nm}^{2}$ ) was calculated using the formula $\left(C_{\text {Sulfur }} / 96\right) /\left(C_{N P S} \times 6 S_{\text {TEM }}{ }^{2}\right) . C_{\text {sulfur }}$ is the concentration of sulfur from ICP in $\mathrm{g} / \mathrm{L}, \mathrm{S}_{\text {TEM }}$ is the average size of the nanoparticles from TEM in nanometers and $C_{N P s}$ is the concentration of nanoparticles (mol nanoparticles/L).

\subsection{Polymerization of NIPAAM, PEGA and NIPAAM/PEGA copolymerization (Example procedure).} SUCRA $(0.130 \mathrm{~g}, 0.35 \mathrm{mmol})$ and AIBN $(0.006 \mathrm{~g}, 0.04 \mathrm{mmol})$ were weighed separately into glass vialsand dissolved in dioxane $(1.0 \mathrm{~mL})$ to make chain transfer agent and initiator stock solutions respectively. The desired amounts of RAFT agent and initiator were then added from the stock solutions to a pre-weighed amount of monomer(s) in a flask containing a stir bar. The solvent was then added to obtain a homogeneous solution with a monomer content of $20 \%(\mathrm{w} / \mathrm{v})$. The reaction vessel was degassed and transferred to a water bath maintained at $60{ }^{\circ} \mathrm{C}$. Polymerization was allowed to proceed for the desired period and the reaction was stopped by quenching in liquid nitrogen and opening the reaction to the atmosphere. Conversions were determined by ${ }^{1} \mathrm{H}$ NMR.

4.9 Functionalization of iron oxide nanocubes with thermo-responsive polymer in viscous solvents. To a glass vial was added NIPAAM (2.5 g, $21.9 \mathrm{mmol})$, SUCRA (2.0 mg, $5.4 \mu \mathrm{mol})$, solvent (diethylene glycol) $(2 \mathrm{~mL})$, and CTCLRA functionalized iron oxide nanocubes ( $0.25 \mathrm{mg}$ Fe, from $0.6 \mu \mathrm{M}$ of iron stock solution) in THF. The solution was vortexed for a few seconds then ultrasonicated at $0{ }^{\circ} \mathrm{C}$ in an ice bath for one 
minute. The vial was sealed with a rubber septum, degassed with nitrogen gas for 30 minutes, and AIBN was then added from a stock solution in dioxane $(25 \mu \mathrm{L}, 3.28 \mu \mathrm{mol})$ via a microliter syringe. Degassing was continued for a further 10 minutes and the reaction contents were vortexed for a few seconds and the vial was placed in a thermostated water bath at $60{ }^{\circ} \mathrm{C}$ for $2 \mathrm{~h}$. The reaction was stopped by placing the vial in an ice bath and opening it to the atmosphere. A sample for analysis by SEC was obtained by taking an aliquot of the reaction mixture (ca. $100 \mathrm{mg}$ ) which was diluted with THF ( $5 \mathrm{~mL}$ ) and placed on a magnet to remove the nanoparticles prior to collection of the supernatant for analysis. The reaction mixture was precipitated into cold diethyl ether and the collected precipitate was dissolved in a small amount of THF and reprecipitated in cold diethyl ether. This process was repeated one more time and the precipitate was then dissolved in THF/diethyl ether (1:2) and centrifuged at $3500 \mathrm{rpm}$ for 10 minutes. If the supernatant was decanted into hexane precipitation of free polymer could be observed. The precipitated nanoparticles were resuspended in THF/diethyl ether (1:2) and centrifuged at 3500 rpm for 10 minutes and this approach was repeated 3 times with the supernatant being poured into hexane. The supernatant from the last wash was concentrated on the rotary evaporator dryness and analyzed by SEC to detect the presence of free polymer. If free polymer was detected, the nanoparticles were washed one more time and the supernatant analysed again by SEC. After the last wash, the nanoparticles were dispersed in $5 \mathrm{~mL}$ of THF and $1 \mathrm{~mL}$ of this solution was transferred to a glass vial which was placed on a magnet to precipitate the particles and the supernatant was filtered, concentrated to $0.3 \mathrm{~mL}$ by evaporation using a slow flow of nitrogen gas and subjected to analysis by SEC to verify the absence of free polymer in the sample (DRI/UV $306 \mathrm{~nm}$ ).

4.10 Cell studies: Viability and Uptake Assay. $5 \times 10^{5} \mathrm{~Kb}$ cells were seeded into 12 wells multiwell plates $24 \mathrm{~h}$ prior the treatment started. A dispersion of PNIPAAM-Co-PEGA functionalized IONPs in PBS at a concentration of $2.1 \mathrm{~g} / \mathrm{L}$ of iron was diluted in 10\% FBS supplemented RPMI, in order to reach a concentration of $1 \mathrm{~g} / \mathrm{L}$ of iron. As controls, cells under the same conditions were left untreated or also were incubated with the same ratio PBS/medium. After $16 \mathrm{~h}$ of incubation, the cells were rinsed 5 times with PBS, and an aliquot of cell dishes were re-incubated with fresh medium for further 32 hours and then analyzed, while other cell wells were directly analyzed under the microscope. For the viability trypan blue assay, after cell trypsinization and washing steps for 3 more times by centrifugation in 10\% FBS supplemented RPMI, all the washing solutions were centrifuged in order to recovery the dead cells eventually detached present in the media. Then, all the cells were mixed together and resuspended in $500 \mu \mathrm{L}$ of medium. $10 \mu \mathrm{L}$ of this cell suspension was mixed with $10 \mu \mathrm{L}$ of trypan blue solution, incubated 
for $20 \mathrm{~min}$ and analyzed in the Burker chamber to count the number of dead and alive cells. $100 \mu \mathrm{L}$ of the cell solution, corresponding to $125,7 \times 10^{3}$ cells were dissolved in aqua regia and the iron contents were estimated by ICP-AES measurements. All measures have been performed in triplicate.

\subsection{Drug loading and release}

(a) Drug loading. To polymer functionalized nanoparticles ( $2 \mathrm{~mL}, 50 \mathrm{ppm}$ of Fe) in PBS buffer (pH 7.4) was added doxorubicin hydrochloride $(150 \mu \mathrm{g})$. The solution was placed on an orbital shaker for 24 hours $(1000 \mathrm{rpm})$ to load the drug within the polymer layer. The unloaded drug was removed by placing the solutions on a magnet whereupon the loaded nanoparticles sedimented and the free drug remained in solution and was drawn off using a micropipette. Fresh PBS buffer was then added to the vial with the nanoparticles in order to re-suspend them and more unloaded drug was then removed as previously described. The process was repeated (usually three to four times) until no absorption signal due to doxo in the supernatant was observed via UV spectroscopy $\left(\lambda_{\max } 485 \mathrm{~nm}\right)$. For the sample used to demonstrate drug release under AMF, the amount of doxo loaded for $6 \mathrm{mg}$ of iron was 2,2 $\mathrm{mg}$ which corresponds to $23 \%$ of doxo loading.

\subsection{Drug release:}

(i) Drug release in thermostated water bath. A colloidal solution of the drug loaded hybrid nanoparticles ( $2 \mathrm{~mL}, 200 \mathrm{ppm} \mathrm{Fe}$ ) was placed in a thermostated water bath at a desired temperature (main text, figure $3 \mathrm{~A}$ ) and at desired intervals, the samples were placed on a magnet for 5 minutes to attract the hybrid nanoparticles and the supernatant was analyzed by UV spectroscopy to determine the amount released using the Beer-Lambert-Law. After each absorption measurement, the supernatant was transferred back into the original vial and the nanoparticles were completely re-dissolved and then incubated again in the water bath.

(ii) Drug release under an alternating magnetic field. Two samples of FeO-(PNIPAAM-Co-PEGA) nanocubes loaded with doxo with an iron concentration of $3.8 \mathrm{~g} / \mathrm{L}(1 \mathrm{~mL})$ in a glass vials were placed on a magnet (0.3T) to remove any traces of unbound/leaked doxorubicin. The collected nanoparticles were re-suspended in $1 \mathrm{~mL}$ of fresh PBS buffer $(\mathrm{pH} \mathrm{7.4)}$ and then one sample was placed centrally in the magnetic coil of the instrumentfollowed by application of an alternating magnetic field whilst theother sample was left at room temperature. The AMF was initially applied for 15 minutes $\left(220 \mathrm{kHz}, 20 \mathrm{kAm}^{-1}\right)$ with the temperature measuring probe dipped in solution. The sample was then taken out of the 
instrument and placed on the magnet for 5 minutes to collect the nanoparticles at the bottom of the vessel. The record of the UV-VIS absorption spectra on the supernatant allows for the measurement of the amount of drug released. After analysis of the supernatant, it was returned back into the vial containing the thermo-responsive coated nanoparticles and completely re-mixed before another cycle of AMF was applied for the desired period. At the next time point, the separation was reiterated and the amount released was again measuredby UV-vis spectroscopy. An aliquot of the doxo loaded sample was kept at room temperature and treated in the same way (but not subjected to an AMF) for comparison. No unspecific release was measured on this sample (see $\mathrm{SI}$ ).

\subsection{Functionalization of superparamagnetic spherical nanoparticles (spherical-IONPs) with catechol bearing RAFT agent (CTCLRA).}

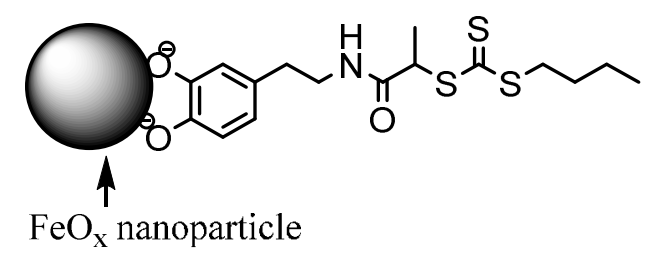

Scheme 5: Spherical-IONPs functionalized CTCLRA.

To a toluene solution of oleic acid capped spherical IONPs $(4 \mathrm{~mL}, 0.6 \mu \mathrm{M}$, [Fe] $1.8 \mathrm{mg} / \mathrm{mL}, 14 \mathrm{~nm}$ diameter(TEM)) was added a THF solution of CTCLRA agent (24 mmol in $45 \mathrm{~mL}$ THF) and then a triethylamine ( $3 \mathrm{~mL}$ in $10 \mathrm{~mL}$ THF) was added. The resulting colloidal solution was left on a shaker overnight after which the nanoparticles were recovered by centrifugation at $4000 \mathrm{rpm}$ for 10 minutes. The collected nanoparticles were redispersed in THF and nanoparticles were washed by the excess of CTCLRA by centrifugation and re-dispersion: before centrifugation hexane was added to the vicinity of particle precipitation (the amount of hexane added was such that no particle precipitation was observed but further addition of hexane (few $\mathrm{mL}$ ) would result in precipitation). The solution was centrifuged at $4000 \mathrm{rpm}$ for 10 minutes to recover the nanoparticles and the supernatant solution was decanted. This process was repeated until the characteristic yellow color of the RAFT agent in the supernatant disappeared (3-4 times) after which the nanoparticles were washed three more times. The functionalized nanoparticles were insoluble in hexane which is a good solvent for the starting nanoparticles, thus indicating a change at the NP surface.ICP (starting nanoparticles), [Fe]= $1.8 \mathrm{~g} / \mathrm{L},[\mathrm{S}]=$ 0. ICP (CTCLRA functionalized nanoparticles) $[\mathrm{Fe}]=1.79 \mathrm{~g} / \mathrm{L},[\mathrm{S}]=0.14 \mathrm{~g} / \mathrm{L}$ (based on same volume of 
starting and CTA functionalized nanoparticles). FTIR: C=S $1061 \mathrm{~cm}^{-1}, \mathrm{~N}-\mathrm{H} 3450 \mathrm{~cm}^{-1}$. Initiator grafting density $\left(9\right.$ molecules $/ \mathrm{nm}^{2}$ ) was estimated using the formula $\left(C_{\text {sulfur }} / 96\right) /\left(C_{\text {NPS }} \times 4 \pi R_{\text {TEM }}{ }^{2}\right)$. $C_{\text {sulfur }}$ is the concentration of sulfur from ICP in $\mathrm{g} / \mathrm{L}, \mathrm{R}_{\mathrm{TEM}}$ is the calculated radius of the nanoparticles from TEM in nanometers and $\mathrm{C}_{\mathrm{NPS}}$ is the concentration of nanoparticles (mol nanoparticles/L).

\subsection{Functionalization of superparamagnetic spherical nanoparticles (spherical IONPs) with thermo- responsive polymer.}

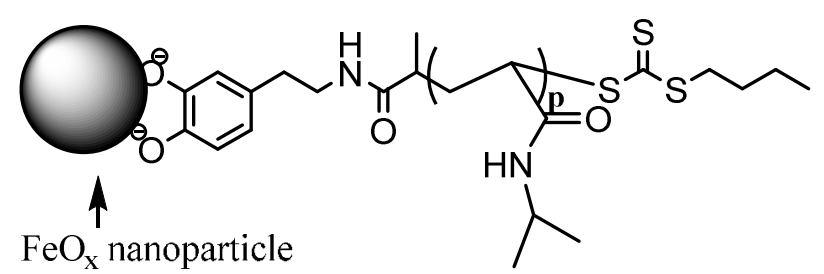

Scheme 6: Spherical-IONPs functionalized with thermo-responsive polymer (PNIPAAM).

To a glass vial was added NIPAAM (4.000 g, $35.4 \mathrm{mmol})$, SUCRA $(0.011 \mathrm{~g}, 32.8 \mu \mathrm{mol})$, solvent (dioxane or THF) (4 g), and CTCLRA-iron oxide nanoparticles dissolved in THF (1 mg Fe, from 0.6 $\mu \mathrm{M}$ stock solution of NPs). The solution was vortexed for a few seconds and the vial was sealed with a rubber septum followed by degassing with nitrogen gas for 30 mins. AIBN ( $25 \mu \mathrm{L}, 3.28 \mu \mathrm{mol}$ ) was then added from a stock solution in dioxane via a microliter syringe and degassing was continued for a further 10 minutes. The reaction contents were mixed for a few seconds and the vial was placed in a thermo-stated water bath at $60{ }^{\circ} \mathrm{C}$ for $3 \mathrm{~h}$. The reaction was stopped by placing the vial in an ice bath and opening it to the atmosphere. A sample for analysis by SEC was obtained by taking an aliquot of the reaction mixture (ca. $100 \mathrm{mg}$ in around $100 \mu \mathrm{L}$ of the sample) which was diluted with THF and placed on a magnet to remove the nanoparticles prior to collection of the supernatant for analysis. The reaction mixture was precipitated into cold diethyl ether and the collected precipitate was dissolved in a small amount of THF and reprecipitated in cold diethyl ether. This process was repeated one more time and the precipitate was then dissolved in THF/diethyl ether (1:2 in volume) and centrifuged at 5000 rpm for 10 minutes. The supernatant was decanted into cold hexane were precipitation of free polymer could be observed. The precipitated nanoparticles were resuspended in THF/diethyl ether (1:2) and centrifuged again at 5000 rpm for 10 minutes and this approach was repeated 3 times with the supernatant being poured into cold hexane. The supernatant from the last wash was concentrated on the rotary evaporator and analyzed by SEC to confirm the absence of free polymer. 


\section{Characterization techniques}

4.15 Nuclear Magnetic Resonace (NMR): NMR analyses were done using Bruker Ultra Shield Avance spectrometers $400 \mathrm{MHz}$ ). For all NMR analyses, unless stated otherwise, deuterated chloroform ( $\mathrm{CDCl} 3)$ was used as the solvent with tetramethylsilane (TMS) as the internal standard.

4.16 Size exclusion chromatography (SEC): SEC was used to determine the molecular weight of the polymers. SEC analyses were carried out at $60 \circ \mathrm{C}$ using an Agilent SEC system equipped with a guard column and two Agilent PolarGel M columns (molecular weight range of 500-2 $000000 \mathrm{~g} / \mathrm{mol}$ ) attached to a differential refractive index (DRI) detector. The flow rate of the system was set at $1 \mathrm{~mL} / \mathrm{min}$ and the eluent was DMF with $0.3 \%(\mathrm{w} / \mathrm{v}) \mathrm{LiBr}$. The SEC system was calibrated using Agilent narrow molecular weight distribution polystyrene standards.

4.17 Dynamic Light scattering (DLS): Particle size measurements were carried out by dynamic light scattering (DLS) using a Malvern Instruments Zetasizernano series instrument. An equilibration time of 3 minutes was allowed before each measurement and at least three replicate measurements were made for each sample ([Fe] = ca. 25 ppm).

4.18 Zeta potential measurements: Zeta potential measurements were carried out using a Malvern Instruments Zetasizernano series instrument. An equilibration time of 3 minutes was allowed before each measurement and at least five replicate measurements were made for each sample ([Fe] $=$ ca. 25 ppm).

4.19 Transmission electron microscopy (TEM): Conventional TEM images were obtained using JEOL JEM 1011 electron microscope, working with an acceleration voltage of $100 \mathrm{kV}$ and equipped with a W thermionic electron source and a 11Mp Orius CCD Camera (Gatan company, USA). Samples were prepared by placing a drop of sample onto a carbon coated copper grid which was then left to dry before imaging. Frozen hydrated samples were prepared by applying a $3 \mu \mathrm{l}$ aliquot to a previously glowdischarged 200-mesh Quantifoil holey carbon grid (Ted Pella, USA). Before plunging the grid into liquid ethane, the grid was blotted for $1.5 \mathrm{~s}$ in a chamber at $4^{\circ} \mathrm{C}$ and $90 \%$ humidity using a FEI Vitrobot Mark IV (FEl company, the Netherlands). The particles were imaged, in both cryo-TEM and cryo-STEM mode, using a Jeol JEM 2200FS (Jeol, Japan) and a Tecnai G2 F20 microscopes (FEI company, the Netherlands), respectively. Both the microscopes were equipped with a Schottky Field Emission electron source, a 
US1000 2kx2k Gatan CCD camera and operated at an acceleration voltage of $200 \mathrm{kV}$. The cryo-TEM imaging was carried out under low dose condition in order to limit water sublimation from the vitrified sample and its recrystallization on the specimen surface.

4.20 Fourier transform infra-red (FT-IR): FT-IR spectra were recorded using a with a Bruker vertex 70v Fourier transform infrared spectrometer. Samples were prepared by placing a drop of sample onto a silicon wafer and left to dry in a desiccator at room temperature overnight before analysis.

4.21 Turbidimetric analysis: Turbidimetric analyses were done using a Varian Cary 5000 UV-vis spectrophotometer equipped with Peltier elements for temperature control. Measurements were done on solutions of ca. $50 \mathrm{ppm}(\mathrm{Fe}$ ) and before each measurement, the sample was left to equilibrate at the desired temperature for 3 minutes.

4.22 pH measurements: Measurements were done using a calibrated Crison Basic $20 \mathrm{pH}$ meter.

4.23 TGA analysis: The weight loss of the functionalised nanoparticles was determined by using a TA Instruments Hi-Res TGA 2950 thermogravimetric analyzer under a nitrogen atmosphere $\left(60 \mathrm{~cm}^{3} / \mathrm{min}\right)$. The samples $(2-3 \mathrm{mg})$ of the thermo-responsive coated nanocubes were heated from room temperature to $50{ }^{\circ} \mathrm{C}$ and isothermal for $15 \mathrm{mins}$ then to $700^{\circ} \mathrm{C}$ with the heating rate set at $5^{\circ} \mathrm{C} / \mathrm{min}$.

4.24 Elemental analysis: Elemental analysis was carried out via Inductively Coupled Plasma (ICP) Atomic Emission Spectroscopy on a ThermoFisher CAP 6000 series. The samples were prepared by digesting $25 \mu \mathrm{L}$ of sample in $2 \mathrm{~mL}$ of aqua regia overnight followed by dilution with milliQ water to 25 $\mathrm{mL}$.

4.25 Magnetic characterization: Magnetic characterization was carried out by using a superconducting quantum interference device (MPMS SQUID) from Quantum Design. Magnetization curves were measured from -80 to $80 \mathrm{kOe}$ at $5 \mathrm{~K}$ and $298 \mathrm{~K}$ upon zero field cooling (ZFC). Zero field cool (ZFC) and field cool (FC) curves were recorded to measure the thermal dependence of the magnetization. Samples were prepared by drop casting an IONPs solution onto a Teflon tape. After evaporation, the final powder was enwrapped and measured. The amount of iron was determined by elemental analysis (ICP). 
4.26 Drug release under an alternating magnetic field (AMF): The hyperthermia experiment was conducted using a NanoscaleBiomagnetics instrument (DM100 series) equipped with a temperaturemeasuring probe. The AMF was set at $220 \mathrm{kHz}, 20 \mathrm{kAm}^{-1}$.

Supporting Information Available: ${ }^{1}$ NMR characterization of succinimide RAFT agent, DLS analysis of poly (NIPAAM) functionalized IONPs in standard media, FTIR spectra and thermogravimetric analysis of nanocube sampels before and after the CTCLRA functionalization, UV-vis spectra of doxo release at various times in the absence of an AMF (room temperature) from NIPAAM-co-PEGA functionalized cubes, characterization of nanocubes having pH-responsive shell as PVP or PVP-co-PEGA and turbidimetric analysis of thermo-responsive spherical-IONP have been added in the supporting information. This information is available free of charge via the Internet at http://pubs.acs.org/.

\section{Acknowledgments}

The authors are grateful for financial support from the European project Magnifyco (Contract NMP4-SL2009-228622) by the Italian AIRC project (contract n. 14527 to TP), EU-ITN network Mag(net)icFun (PITN-GA-2012-290248) and by the Italian FIRB projects (Nanostructured oxides, contract no.588 BAP115AYN).

\section{References}

1. Laurent, S.; Forge, D.; Port, M.; Roch, A.; Robic, C.; Vander Elst, L.; Muller, R. N. Magnetic Iron Oxide Nanoparticles: Synthesis, Stabilization, Vectorization, Physicochemical Characterizations, and Biological Applications. Chem. Rev. 2008, 108, 2064-2110.

2. Gupta, A. K.; Gupta, M. Synthesis and Surface Engineering of Iron Oxide Nanoparticles for Biomedical Applications. Biomaterials 2005, 26, 3995-4021. 
3. Jun, Y.-W.; Lee, J.-H.; Cheon, J. Chemical Design of Nanoparticle Probes for High-Performance Magnetic Resonance Imaging. Angew. Chem. Int. Ed. 2008, 47, 5122-5135.

4. Amstad, E.; Gillich, T.; Bilecka, I.; Textor, M.; Reimhult, E. Ultrastable Iron Oxide Nanoparticle Colloidal Suspensions Using Dispersants with Catechol-Derived Anchor Groups. Nano Lett. 2009, 9, 40424048.

5. Neoh, K. G.; Kang, E. T. Functionalization of Inorganic Nanoparticles with Polymers for Stealth Biomedical Applications. Polym. Chem. 2011, 2, 747-759.

6. Quarta, A.; Curcio, A.; Kakwere, H.; Pellegrino, T. Polymer Coated Inorganic Nanoparticles: Tailoring the Nanocrystal Surface for Designing Nanoprobes with Biological Implications. Nanoscale 2012, 4, 3319-3334.

7. Boyer, C.; Bulmus, V.; Priyanto, P.; Teoh, W. Y.; Amal, R.; Davis, T. P. The Stabilization and BioFunctionalization of Iron Oxide Nanoparticles Using Heterotelechelic Polymers. J. Mater. Chem. 2009, $19,111-123$.

8. Guo, Y.; Harirchian-Saei, S.; Izumi, C. M. S.; Moffitt, M. G. Block Copolymer Mimetic SelfAssembly of Inorganic Nanoparticles. ACS Nano 2011, 5, 3309-3318.

9. Boyer, C.; Whittaker, M. R.; Bulmus, V.; Liu, J. Q.; Davis, T. P. The Design and Utility of PolymerStabilized Iron-Oxide Nanoparticles for Nanomedicine Applications. NPG Asia Mater. 2010, 2, 23-30.

10. Oh, J. K.; Park, J. M. Iron Oxide-Based Superparamagnetic Polymeric Nanomaterials: Design, Preparation, and Biomedical Application. Prog. Polym. Sci. 2011, 36, 168-189.

11. Beija, M.; Marty, J. D.; Destarac, M. Raft/Madix Polymers for the Preparation of Polymer/Inorganic Nanohybrids. Prog. Polym. Sci. 2011, 36, 845-886.

12. Matyjaszewski, K.; Davis, T. P., Handbook of Radical Polymerization. Wiley - Interscience New Jersey, 2002. 
13. Chiefari, J.; Chong, Y. K.; Ercole, F.; Krstina, J.; Jeffery, J.; Le, T. P. T.; Mayadunne, R. T. A.; Meijs, G. F.; Moad, C. L.; Moad, G.; Rizzardo, E.; Thang, S. H. Living Free-Radical Polymerization by Reversible Addition-Fragmentation Chain Transfer: The Raft Process. Macromolecules 1998, 31, 5559-5562.

14. Louguet, S.; Rousseau, B.; Epherre, R.; Guidolin, N.; Goglio, G.; Mornet, S.; Duguet, E.; Lecommandoux, S.; Schatz, C. Thermoresponsive Polymer Brush-Functionalized Magnetic Manganite Nanoparticles for Remotely Triggered Drug Release. Polym. Chem. 2012, 3, 1408-1417.

15. Purushotham, S.; Ramanujan, R. V. Thermoresponsive Magnetic Composite Nanomaterials for Multimodal Cancer Therapy. Acta Biomater. 2010, 6, 502-510.

16. Aqil, A.; Vasseur, S.; Duguet, E.; Passirani, C.; Benoit, J. P.; Jerome, R.; Jerome, C. Magnetic Nanoparticles Coated by Temperature Responsive Copolymers for Hyperthermia. J. Mater. Chem. 2008, $18,3352-3360$.

17. Liu, J.; Detrembleur, C.; Debuigne, A.; De Pauw-Gillet, M.-C.; Mornet, S.; Vander Elst, L.; Laurent, S.; Labrugere, C.; Duguet, E.; Jerome, C. Poly(Acrylic Acid)-Block-Poly(Vinyl Alcohol) Anchored Maghemite Nanoparticles Designed for Multi-Stimuli Triggered Drug Release. Nanoscale 2013, 5, 1146411477.

18. Hayashi, K.; Nakamura, M.; Miki, H.; Ozaki, S.; Abe, M.; Matsumoto, T.; Sakamoto, W.; Yogo, T.; Ishimura, K. Magnetically Responsive Smart Nanoparticles for Cancer Treatment with a Combination of Magnetic Hyperthermia and Remote-Control Drug Release. Theranostics 2014, 4, 834-844.

19. Deka, S. R.; Quarta, A.; Di Corato, R.; Riedinger, A.; Cingolani, R.; Pellegrino, T. Magnetic Nanobeads Decorated by Thermo-Responsive Pnipam Shell as Medical Platforms for the Efficient Delivery of Doxorubicin to Tumour Cells. Nanoscale 2011, 3, 619-629.

20. Riedinger, A.; Pernia Leal, M.; Deka, S. R.; George, C.; Franchini, I. R.; Falqui, A.; Cingolani, R.; Pellegrino, T. "Nanohybrids" Based on pH-Responsive Hydrogels and Inorganic Nanoparticles for Drug Delivery and Sensor Applications. Nano Lett. 2011, 11, 3136-3141. 
21. Guardia, P.; Riedinger, A.; Nitti, S.; Pugliese, G.; Marras, S.; Genovese, A.; Materia, M. E.; Lefevre, C.; Manna, L.; Pellegrino, T. One Pot Synthesis of Monodisperse Water Soluble Iron Oxide Nanocrystals with High Values of the Specific Absorption Rate. J. Mater. Chem. B 2014.

22. Guardia, P.; Di Corato, R.; Lartigue, L.; Wilhelm, C.; Espinosa, A.; Garcia-Hernandez, M.; Gazeau, F.; Manna, L.; Pellegrino, T. Water-Soluble Iron Oxide Nanocubes with High Values of Specific Absorption Rate for Cancer Cell Hyperthermia Treatment. ACS Nano 2012, 6, 3080-3091.

23. Kolosnjaj-Tabi, J.; Di Corato, R.; Lartigue, L.; Marangon, I.; Guardia, P.; Silva, A. K. A.; Luciani, N.; Clément, O.; Flaud, P.; Singh, J. V.; Decuzzi, P.; Pellegrino, T.; Wilhelm, C.; Gazeau, F. Heat-Generating Iron Oxide Nanocubes: Subtle "Destructurators" of the Tumoral Microenvironment. ACS Nano 2014, 8, 4268-4283.

24. Lartigue, L.; Alloyeau, D.; Kolosnjaj-Tabi, J.; Javed, Y.; Guardia, P.; Riedinger, A.; Péchoux, C.; Pellegrino, T.; Wilhelm, C.; Gazeau, F. Biodegradation of Iron Oxide Nanocubes: High-Resolution in Situ Monitoring. ACS Nano 2013, 7, 3939-3952.

25. Riedinger, A.; Guardia, P.; Curcio, A.; Garcia, M. A.; Cingolani, R.; Manna, L.; Pellegrino, T. Subnanometer Local Temperature Probing and Remotely Controlled Drug Release Based on AzoFunctionalized Iron Oxide Nanoparticles. Nano Letters 2013, 13, 2399-2406.

26. Ohno, K.; Ma, Y.; Huang, Y.; Mori, C.; Yahata, Y.; Tsujii, Y.; Maschmeyer, T.; Moraes, J.; Perrier, S. Surface-Initiated Reversible Addition-Fragmentation Chain Transfer (Raft) Polymerization from Fine Particles Functionalized with Trithiocarbonates. Macromolecules 2011, 44, 8944-8953.

27. Aseyev, V.; Tenhu, H.; Winnik, F., Non-lonic Thermoresponsive Polymers in Water. In Self Organized Nanostructures of Amphiphilic Block Copolymers li, Müller, A. H. E.; Borisov, O., Eds. Springer Berlin Heidelberg: 2011; Chapter 57, pp 29-89.

28. Melville, H. W.; Murray, A. J. R. The Ultrasonic Degradation of Polymers. Trans. Faraday Soc. 1950, 46, 996-1009. 
29. Price, G. J.; Smith, P. F. Ultrasonic Degradation of Polymer Solutions. 1. Polystyrene Revisited. Polym. Int. 1991, 24, 159-164.

30. West, A. G.; Barner-Kowollik, C.; Perrier, S. Poly(ethylene glycol) as a 'Green Solvent' for the Raft Polymerization of Methyl Methacrylate. Polymer 2010, 51, 3836-3842.

31. Perrier, S.; Gemici, H.; Li, S. Poly(ethylene glycol) as Solvent for Transition Metal Mediated Living Radical Polymerisation. Chem. Commun. (Cambridge, U. K.) 2004, 0, 604-605.

32. Kumar, C. S. S. R.; Mohammad, F. Magnetic Nanomaterials for Hyperthermia-Based Therapy and Controlled Drug Delivery. Adv. Drug Delivery Rev. 2011, 63, 789-808.

33. Knop, K.; Hoogenboom, R.; Fischer, D.; Schubert, U. S. Poly(ethylene glycol) in Drug Delivery: Pros and Cons as Well as Potential Alternatives. Angew. Chem. Int. Ed. 2010, 49, 6288-6308.

34. Cullity, B. D. Introduction to Magnetism and Magnetic Materials. Addison-Wesley: Massachusetts Text Book 1972.

35. Yu, W. W.; Falkner, J. C.; Yavuz, C. T.; Colvin, V. L. Synthesis of Monodisperse Iron Oxide Nanocrystals by Thermal Decomposition of Iron Carboxylate Salts. Chem. Commun. (Cambridge, U. K.) 2004, 0, 2306-2307.

36. Ferguson, C. J.; Hughes, R. J.; Nguyen, D.; Pham, B. T. T.; Gilbert, R. G.; Serelis, A. K.; Such, C. H.; Hawkett, B. S. Ab Initio Emulsion Polymerization by Raft-Controlled Self-Assembly§. Macromolecules 2005, 38, 2191-2204.

37. Yu, W. W.; Falkner, J. C.; Yavuz, C. T.; Colvin, V. L. Synthesis of Monodisperse Iron Oxide Nanocrystals by Thermal Decomposition of Iron Carboxylate Salts. Chem. Commun. (Cambridge, U. K.) 2004, 2306-2307. 


\section{Graphic Table of Contents}

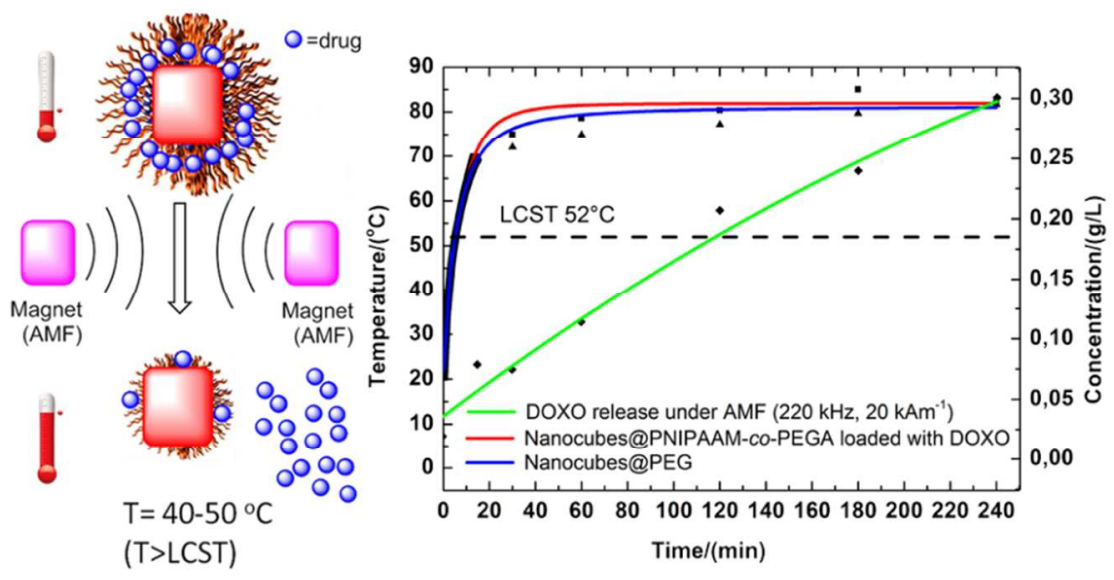

et 